\title{
Optimizing the fundamental Neumann eigenvalue for the Laplacian in a domain with small traps
}

\author{
T. KOLOKOLNIKOV ${ }^{1}$, M. S. TITCOMBE${ }^{2}$ and M. J. WARD W $^{3} \dagger$ \\ ${ }^{1}$ Department of Mathematics, University of British Columbia, Vancouver, Canada V6T $1 Z 2$ \\ ${ }^{2}$ Centre de recherches mathématiques, University of Montreal, Montreal, Canada, H3C 3J7 \\ ${ }^{3}$ Department of Mathematics, University of British Columbia, Vancouver, Canada V6T 1Z2
}

(Received 7 September 2004)

\begin{abstract}
An optimization problem for the fundamental eigenvalue $\lambda_{0}$ of the Laplacian in a planar simply-connected domain that contains $N$ small identically-shaped holes, each of radius $\varepsilon \ll 1$, is considered. The boundary condition on the domain is assumed to be of Neumann type, and a Dirichlet condition is imposed on the boundary of each of the holes. As an application, the reciprocal of the fundamental eigenvalue $\lambda_{0}$ is proportional to the expected lifetime for Brownian motion in a domain with a reflecting boundary that contains $N$ small traps. For small hole radii $\varepsilon$, a two-term asymptotic expansion for $\lambda_{0}$ is derived in terms of certain properties of the Neumann Green's function for the Laplacian. Only the second term in this expansion depends on the locations $x_{i}$, for $i=1, \ldots, N$, of the small holes. For the unit disk, ring-type configurations of holes are constructed to optimize this term with respect to the hole locations. The results yield hole configurations that asymptotically optimize $\lambda_{0}$. For a class of symmetric dumbbell-shaped domains containing exactly one hole, it is shown that there is a unique hole location that maximizes $\lambda_{0}$. For an asymmetric dumbbell-shaped domain, it is shown that there can be two hole locations that locally maximize $\lambda_{0}$. This optimization problem is found to be directly related to an oxygen transport problem in skeletal muscle tissue, and to determining equilibrium locations of spikes to the Gierer-Meinhardt reactiondiffusion model. It is also closely related to the problem of determining equilibrium vortex configurations within the context of the Ginzburg-Landau theory of superconductivity.
\end{abstract}

\section{Introduction}

We consider an optimization problem for the fundamental eigenvalue of the Laplacian in a bounded two-dimensional domain with a reflecting boundary that is perturbed by the presence of $N$ small holes in the interior of the domain. The perturbed eigenvalue problem is

$$
\begin{aligned}
& \Delta u+\lambda u=0, \quad x \in \Omega \backslash \Omega_{p} ; \quad \int_{\Omega \backslash \Omega_{p}} u^{2} d x=1, \\
& \partial_{n} u=0, \quad x \in \partial \Omega ; \quad u=0, \quad x \in \partial \Omega_{p} \equiv \cup_{i=1}^{N} \partial \Omega_{\varepsilon_{i}} .
\end{aligned}
$$

$\dagger$ Corresponding author. 
Here $\Omega$ is the unperturbed domain, $\Omega_{p}=\cup_{i=1}^{N} \Omega_{\varepsilon_{i}}$ is a collection of $N$ small interior holes $\Omega_{\varepsilon_{i}}$, for $i=1, \ldots, N$, each of 'radius' $O(\varepsilon)$, and $\partial_{n} u$ is the outward normal derivative of $u$ on $\partial \Omega$. We assume that the small holes in $\Omega$ are non-overlapping and that $\Omega_{\varepsilon_{i}} \rightarrow x_{i}$ as $\varepsilon \rightarrow 0$, for $i=1, \ldots, N$.

We let $\lambda_{0}(\varepsilon)$ denote the first eigenvalue of (1.1), with corresponding eigenfunction $u(x, \varepsilon)$. Clearly, $\lambda_{0}(\varepsilon) \rightarrow 0$ as $\varepsilon \rightarrow 0$. Our objective is to determine the locations, $x_{i}$ for $i=1, \ldots, N$, of the $N$ holes of a given shape that maximize this fundamental eigenvalue. Asymptotic expansions for the fundamental eigenvalue of related eigenvalue problems in perforated multi-dimensional domains, with various boundary conditions on the holes and outer boundary, are given in [7, 20, 23, 26, 28, 29] (see also the references therein). Optimization problems for the fundamental eigenvalue of the Laplacian in planar domains under strong changes in the boundary conditions from Neumann to Dirichlet have been studied in $[5,6]$. Optimization problems for eigenvalues of the Laplacian involving isoperimetric inequalities have a long history in mathematical physics. For a recent survey see [13].

A related work on optimizing Laplacian eigenvalues in perforated domains is that of [12]. The problem of [12] is concerned with optimizing the fundamental Dirichlet eigenvalue $\lambda_{0 d}$ of the Laplacian in a bounded planar domain that contains a hole, with $u=0$ on the boundary of the hole. The hole, which is not necessarily small, has a fixed circular shape but its centre $x_{0}$ can be chosen to optimize $\lambda_{0 d}$. Under certain symmetry conditions on the domain, it was proved in [12] that $\lambda_{0 d}$ is maximized when $x_{0}$ is at a certain interior symmetry point of the domain, and it is minimized when the hole is in contact with the boundary of the domain. Related results for the case of two holes were also proved in [12]. For a circular hole of radius $\varepsilon \ll 1$ that is centred at some interior point $x_{0}$, it is well-known (cf. $\left.[23,28]\right)$ that $\lambda_{0 d}$ has the two-term expansion

$$
\lambda_{0 d} \sim \lambda_{0 d}^{(0)}+2 \pi v\left[u_{0}\left(x_{0}\right)\right]^{2}+O\left(v^{2}\right), \quad v \equiv-1 / \log \varepsilon .
$$

Here $\lambda_{0 d}^{(0)}$ is the fundamental Dirichlet eigenvalue for the unperturbed domain with normalized eigenfunction $u_{0}(x)$. For a small hole, it is clear from (1.2) that $\lambda_{0 d}$ is maximized for $v \ll 1$ at maximum points of $u_{0}\left(x_{0}\right)$. This result also suggests that for a symmetric dumbbell-shaped domain with a thin neck, for which $u_{0}$ is concentrated in both lobes of the dumbbell, there will be at least two locations where $\lambda_{0 d}$ is maximized.

In contrast to this Dirichlet problem, the problem of optimizing the fundamental eigenvalue $\lambda_{0}$ of (1.1) is a little more subtle. For the case of $N$ circular holes, each of radius $\varepsilon \ll 1$, it is well-known (cf. $[23,28]$ ) that

$$
\lambda_{0}(\varepsilon) \sim \frac{2 \pi N v}{|\Omega|}+O\left(v^{2}\right), \quad v \equiv-1 / \log \varepsilon .
$$

Here $|\Omega|$ is the area of $\Omega$. Since this leading term in $\lambda_{0}(\varepsilon)$ is independent of $x_{1}, \ldots, x_{N}$, it gives no information on how to choose an optimal set of hole locations. In [29] it was shown that the expansion of $\lambda_{0}(\varepsilon)$ starts with an infinite logarithmic series in $\varepsilon$. A hybrid asymptotic-numerical method was formulated in [28] to sum this entire series for a given set of hole locations. Although this approach yields an accurate approximation 
to $\lambda_{0}$, it does not provide an analytical expression from which one can determine the hole locations that maximize $\lambda_{0}$.

In $\S 2$ we use the method of matched asymptotic expansions to derive a new analytical result for the next term in the expansion (1.3). For $N$ identical holes the expansion has the form (see Corollary 2.3 below)

$$
\lambda_{0}(\varepsilon) \sim \frac{2 \pi N v}{|\Omega|}-\frac{4 \pi^{2} v^{2}}{|\Omega|} p\left(x_{1}, \ldots, x_{N}\right)+O\left(v^{3}\right),
$$

where the function $p\left(x_{1}, \ldots, x_{N}\right)$ is defined by

$$
p\left(x_{1}, \ldots, x_{N}\right)=\sum_{j=1}^{N}\left(R_{m}\left(x_{j} ; x_{j}\right)+\sum_{\substack{k=1 \\ k \neq j}}^{N} G_{m}\left(x_{j} ; x_{k}\right)\right) .
$$

Here $G_{m}\left(x ; x_{0}\right)$ is the Neumann Green's function, with regular part $R_{m}\left(x ; x_{0}\right)$, satisfying

$$
\begin{gathered}
\Delta G_{m}=\frac{1}{|\Omega|}-\delta\left(x-x_{0}\right), \quad x \in \Omega ; \quad \partial_{n} G_{m}=0, \quad x \in \partial \Omega, \\
G_{m}\left(x ; x_{0}\right)=-\frac{1}{2 \pi} \log \left|x-x_{0}\right|+R_{m}\left(x ; x_{0}\right) ; \quad \int_{\Omega} G_{m}\left(x ; x_{0}\right) d x=0 .
\end{gathered}
$$

Therefore, for $v \ll 1,(1.4 a)$ shows that $\lambda_{0}$ has a local maximum at a local minimum point of $p\left(x_{1}, \ldots, x_{N}\right)$.

As an application of (1.1), we consider, as in $\S 9$ of [28], the Brownian motion of a particle in a two-dimensional domain $\Omega$, with reflecting walls, that contains $N$ small traps $\Omega_{\varepsilon_{i}}$, for $i=1, \ldots, N$, each of 'radius' $\varepsilon$, for $i=1, \ldots, N$. The traps are centred at $x_{i}$, for $i=1, \ldots, N$. If the Brownian particle starts from the point $y \in \Omega \backslash \Omega_{p}$ at time $t=0$, then the probability density $v(x, y, t, \varepsilon)$ that the particle is at point $x$ at time $t$ satisfies

$$
v_{t}=\Delta v, \quad x \in \Omega \backslash \Omega_{p} ; \quad \partial_{n} v=0, \quad x \in \partial \Omega ; \quad v=0, \quad x \in \partial \Omega_{p} ; \quad v=\delta(x-y), \quad t=0 .
$$

As in (1.1), $\Omega_{p}$ is the union of $N$ small non-overlapping holes. By calculating the solution to (1.6) in terms of an eigenfunction expansion, and by assuming that $y$ is uniformly distributed over $\Omega \backslash \Omega_{p}$, it is easy to show that the probability $P_{0}(t, \varepsilon)$ that the Brownian particle is in $\Omega \backslash \Omega_{p}$ at time $t$ is given by (see equation (9.5) of [28])

$$
P_{0}(t, \varepsilon)=e^{-\lambda_{0}(\varepsilon) t}[1+O(v)]
$$

Therefore, the expected lifetime of the Brownian particle is proportional to $1 / \lambda_{0}(\varepsilon)$. In this context, our optimization problem is equivalent to choosing the locations of $N$ small traps to minimize the expected lifetime of the Brownian particle. No optimization problem of this type was considered in [28].

In $\S 3$ we give some results for the optimization of $\lambda_{0}$ in a dumbbell-shaped domain that contains one hole centred at some point $x_{0}$. From (1.4), local minima of $R_{m}\left(x_{0} ; x_{0}\right)$ correspond to local maxima of $\lambda_{0}$. We investigate whether there is a unique location $x_{0}$ of 
a hole, or trap, that maximizes $\lambda_{0}$ in an arbitrary, possibly non-convex, simply-connected domain. For a small hole size, this problem is equivalent to determining whether or not there is a unique root to $\left.\nabla R_{m 0} \equiv \nabla R_{m}\left(x ; x_{0}\right)\right|_{x=x_{0}}=0$. In the context of determining the equilibrium location for a one-spike solution to the Gierer-Meinhardt reaction-diffusion model (cf. [8]), it was proved in [15] that there is a unique root to $\nabla R_{m 0}=0$ in a class of symmetric dumbbell-shaped domains. This root is located in the thin neck region separating the two lobes of the dumbbell. Based on this result, and further numerical evidence, it was conjectured in [15] that the uniqueness of the root to $\nabla R_{m 0}=0$ holds more generally for simply-connected domains. In $\S 3$, we show that this conjecture is indeed false by constructing a family of asymmetric dumbbell-shaped domains for which $\nabla R_{m 0}=0$ has multiple roots. This counter-example is given in Proposition 3.2. Hence, for an asymmetric dumbbell-shaped domain there can be several trap locations that locally maximize $\lambda_{0}$.

A key open problem, that we do not address, is to determine general properties of $R_{m}\left(x_{0} ; x_{0}\right)$ and $\nabla R_{m 0}$ under various conditions on the domain. In particular, is there a unique root to $\nabla R_{m 0}=0$ in any simply-connected convex domain? If so, then there must be a unique trap location that maximizes $\lambda_{0}$ in such a domain. In contrast, many results are available for the regular part $R_{d}\left(x ; x_{0}\right)$ of the Dirichlet Green's function $G_{d}\left(x ; x_{0}\right)$, satisfying $\Delta G_{d}=-\delta\left(x-x_{0}\right)$ in $\Omega$, with $G_{d}=0$ on $\partial \Omega$. For a survey of such results see [1]. In a planar convex domain, $\nabla R_{d 0}=0$ has a unique root (cf. [3]), and in the class of symmetric dumbbell-shaped domains of [11], which were also used in $[15,16]$, there can be multiple roots to $\nabla R_{d 0}=0$ (cf. [11]).

In $\S 4$ and $\S 5$ we optimize $\lambda_{0}$ for various configurations of identically-shaped holes inside the unit disk. The advantage of considering the unit disk is that the solution to (1.5) is readily available. In $\S 4.1$ we optimize $p\left(x_{1}, \ldots, x_{N}\right)$ in $(1.4 b)$ when $N$ identical holes are located symmetrically on a ring of radius $r$. For this configuration we can calculate the function $p=p(r)$ explicitly. From this formula it is shown that $p(r)$ has a unique minimum in $0<r<1$ at some $r=r_{c}$, which depends on $N$. We then consider a related optimization problem for a different $N$-hole pattern consisting of $N-1$ identical holes located symmetrically on a ring of radius $r$ and with one hole located at the centre of the unit disk. In $\S 5$ we optimize $p$ for a two-ring pattern in the unit disk that has a total of $N$ identical holes. The generalization to an $m$-ring pattern with $m>2$ is also analyzed. Numerical results for the optimal configuration of holes when $N=6, \ldots, 25$, which are obtained by optimizing $p$ with respect to the ring radii, are given. These results for the unit disk, where $p$ is optimized with respect to certain ring radii, are compared with numerical results computed from the routine fminunc of MATLAB for the $2 \mathrm{~N}$ variable optimization problem of minimizing $p$ for arbitrary hole locations within the unit disk.

The results of $\S 4$ and $\S 5$ for the optimal configurations of holes inside the unit disk that maximize $\lambda_{0}$ are qualitatively similar to numerical and experimental results obtained in $[19,24]$, respectively, for ground-state configurations of certain interacting particle systems. These systems are characterized by a balance between an inter-particle repulsive Coulomb force and a global confinement potential. This potential, typically modelled by a simple quadratic form, restricts the particles to some confinement cell (cf. [19, 24]). The optimization of $p$ in $(1.4 b)$ has the same structure in that the decomposition $(1.5 b)$ shows that $G_{m}$ is the sum of a pure Coulomb singularity and a 'confinement potential', $R_{m}$, that is 
bounded inside $\Omega$. An optimization problem of this type for Coulombic interactions and a logarithmic confinement potential was analyzed rigorously in [9]. As we discuss in $\S 6$, the optimization of $p\left(x_{1}, \ldots, x_{N}\right)$ is also closely related to the problem of minimizing a certain renormalized energy associated with the Ginzburg-Landau model of superconductivity. Minimum points of this energy correspond to equilibrium configurations of vortices.

In $\S 6$ we show that the problem of minimizing $p\left(x_{1}, \ldots, x_{N}\right)$ in (1.4) also arises in two other distinct contexts. The first problem concerns maximizing the average oxygen partial pressure in a two-dimensional cross-section of muscle tissue that contains $N$ identical capillary cross-sections of small area. A mathematical model for this problem is given in [27]. The second problem concerns the determination of the equilibrium locations of an $N$-spike solution to the singularly perturbed Gierer-Meinhardt reaction-diffusion model (cf. [8]) in a bounded two-dimensional domain. In a certain asymptotic regime, the points where $p\left(x_{1}, \ldots, x_{N}\right)$ has a local minimum correspond to equilibrium spike locations. A few conclusions and open problems are given in $\S 7$.

\section{Determining a system for the fundamental eigenvalue}

We first consider (1.1) for the case of one hole. In [29] it was shown that as $\varepsilon \rightarrow 0$ the first eigenvalue $\lambda_{0}$ of (1.1) has the asymptotic expansion:

$$
\lambda_{0}(\varepsilon)=\lambda_{00}+v(\varepsilon) \lambda_{01}+v^{2}(\varepsilon) \lambda_{02}+\cdots .
$$

Here, $v(\varepsilon)=-1 / \log (\varepsilon d)$ where $d$ is a constant that is determined by the shape of the perturbing hole. For the unperturbed problem with $\varepsilon=0$, we have $\lambda_{00}=0$. In the $O(v)$ term, $\lambda_{01}$ is independent of the position of the hole at $x=x_{0}$. Thus, we need higher-order terms to determine the location of the hole that maximizes the first eigenvalue, $\lambda_{0}$. An infinite logarithmic expansion for $\lambda_{0}(\varepsilon)$ has the form

$$
\lambda_{0}(\varepsilon)=\lambda^{*}(v)+O\left(\frac{\varepsilon}{\log \varepsilon}\right), \quad v \equiv-\frac{1}{\log (\varepsilon d)} .
$$

In [28] it was shown how to formulate an equation for $\lambda^{*}(v)$.

To calculate $\lambda^{*}(v)$ we use the method of matched asymptotic expansions as in [28]. Near the hole, we identify an inner (local) region in terms of a local spatial variable $y=\varepsilon^{-1}\left(x-x_{0}\right)$, and where the hole is rescaled so that $\Omega_{\varepsilon}=\varepsilon \Omega_{0}$. Denoting the inner (local) solution by $v(y, \varepsilon)=u\left(x_{0}+\varepsilon y, \varepsilon\right)$, we then expand $v(y, \varepsilon)$ as

$$
v(y, \varepsilon)=A v v_{c}(y)+\cdots .
$$

Here, $A=A(v) \sim O(1)$ as $\varepsilon \rightarrow 0$, and $v_{c}(y)$ is chosen to be the inner (local) solution with logarithmic behaviour at infinity, which satisfies

$$
\begin{gathered}
\Delta_{y} v_{c}=0, \quad y \notin \Omega_{0} ; \quad v_{c}=0, \quad y \in \partial \Omega_{0}, \\
v_{c} \sim \log |y|-\log d+\frac{p \cdot y}{|y|^{2}}, \quad y \rightarrow \infty .
\end{gathered}
$$


In $(2.2 b)$, the constant $d$ and the dipole vector $p=\left(p_{1}, p_{2}\right)$ are determined from the shape of the hole.

We expand the eigenvalue $\lambda_{0}$ and the outer (global) solution as

$$
\lambda_{0}(\varepsilon)=\lambda^{*}(v)+\mu \lambda_{1}+\cdots, \quad u(x, \varepsilon)=u^{*}(x, v)+\mu u_{1}(x, v)+\cdots,
$$

where $\mu \ll(-1 / \log \varepsilon)^{m}$ for any $m>0$. Substituting (2.3) into $(1.1 a)$ and the boundary condition $(1.1 b)$ on $\partial \Omega$, we obtain the full problem in a domain punctured by the point $x_{0}$,

$$
\Delta u^{*}+\lambda^{*} u^{*}=0, \quad x \in \Omega \backslash\left\{x_{0}\right\} ; \quad \int_{\Omega}\left(u^{*}\right)^{2} d x=1 ; \quad \partial_{n} u^{*}=0, \quad x \in \partial \Omega .
$$

The 'missing' condition on $u^{*}$ is a singularity condition as $x \rightarrow x_{0}$ that comes from matching $u^{*}$ to the local solution. Substituting (2.2 b) into (2.1), and expressing the result in global variables, we obtain

$$
v(y, \varepsilon) \sim A v \log \left|x-x_{0}\right|+A+\varepsilon A v \frac{p \cdot\left(x-x_{0}\right)}{\left|x-x_{0}\right|^{2}}+\cdots, \quad y \rightarrow \infty .
$$

Here, we have used $v \equiv-1 / \log (\varepsilon d)$. To match $u^{*}$ to $(2.5)$, we require that $u^{*}$ has the singularity behaviour

$$
u^{*}(x, \varepsilon) \sim A v \log \left|x-x_{0}\right|+A, \quad x \rightarrow x_{0} .
$$

Comparing the terms in (2.5) and (2.3) at the next order, we see that $\mu=O(\varepsilon v)$.

Next, we determine $u^{*}(x, v)$ and $\lambda^{*}(v)$ satisfying (2.4) and (2.6). To do so, we introduce the Green's function, $G\left(x ; x_{0}, \lambda^{*}\right)$, for the Helmholtz operator, and its regular part, $R\left(x ; x_{0}, \lambda^{*}\right)$, satisfying

$$
\begin{gathered}
\Delta G+\lambda^{*} G=-\delta\left(x-x_{0}\right), \quad x \in \Omega ; \quad \partial_{n} G=0, \quad x \in \partial \Omega, \\
G\left(x ;, x_{0}, \lambda^{*}\right)=-\frac{1}{2 \pi} \log \left|x-x_{0}\right|+R\left(x ; x_{0}, \lambda^{*}\right) .
\end{gathered}
$$

In terms of this Green's function, $u^{*}(x, v)$ is given by

$$
u^{*}(x, v)=-2 \pi A v G\left(x ; x_{0}, \lambda^{*}\right)
$$

By using $(2.7 b)$, we expand $u^{*}$ as $x \rightarrow x_{0}$ to obtain

$$
u^{*}(x, v) \sim A v \log \left|x-x_{0}\right|-2 \pi A v R\left(x_{0} ; x_{0}, \lambda^{*}\right), \quad x \rightarrow x_{0} .
$$

The matching condition is that the expressions in (2.6) and (2.8) agree. The $\log \left|x-x_{0}\right|$ terms automatically agree, and from the remaining terms, we obtain a transcendental equation for $\lambda^{*}(v)$ :

$$
R\left(x_{0} ; x_{0}, \lambda^{*}\right)=-\frac{1}{2 \pi v} .
$$

To obtain the asymptotic behaviour for $\lambda_{0}$, we need the solution $\lambda^{*}$ of (2.9) that tends to zero as $v \rightarrow 0$. 
Equation (2.9) can, in general, only be solved numerically as a function of $v$. Below, we only determine an expression for $\lambda^{*}$ that is correct to terms of order $O\left(v^{2}\right)$. To obtain this expression, we expand the Helmholtz Green's function, $G\left(x ; x_{0}, \lambda^{*}\right)$, in terms of $\lambda^{*} \ll 1$, as

$$
G\left(x ; x_{0}, \lambda^{*}\right)=\frac{1}{\lambda^{*}} G_{0}\left(x ; x_{0}\right)+G_{1}\left(x ; x_{0}\right)+\lambda^{*} G_{2}\left(x ; x_{0}\right)+\cdots
$$

Substituting (2.10) into (2.7), we get a series of problems for the $G_{j}\left(x ; x_{0}\right), j=0,1,2, \ldots$ At order $O\left(1 / \lambda^{*}\right), G_{0}$ satisfies $\Delta G_{0}=0$ in $\Omega$ and $\partial_{n} G_{0}=0$ on $\partial \Omega$, from which we obtain that $G_{0}$ is a constant. The higher-order corrections $G_{j}$ for $j \geqslant 1$ are readily found to satisfy

$$
\begin{gathered}
\Delta G_{j}=\left\{\begin{array}{ll}
-\delta\left(x-x_{0}\right)-G_{0}, & j=1, \\
-G_{j-1}, & j>1,
\end{array} \quad x \in \Omega ;\right. \\
\partial_{n} G_{j}=0, \quad x \in \partial \Omega, \quad j \geqslant 1 ; \quad \int_{\Omega} G_{j} d x=0, \quad j \geqslant 1 .
\end{gathered}
$$

Applying the Divergence Theorem, we obtain that $G_{0}=-1 /|\Omega|$, where $|\Omega|$ is the area of $\Omega$. The function $G_{1}\left(x ; x_{0}\right)$ (which we shall henceforth call $G_{m}$ ) satisfies $(1.5)$ of $\S 1$. The function $G_{m}\left(x ; x_{0}\right)$ is called the Neumann Green's function, or the modified Green's function, and $R_{m}\left(x ; x_{0}\right)$ is called the regular part of $G_{m}\left(x ; x_{0}\right)$.

From (2.10) and (1.5b), we write the two-term expansion for $G$ when $\lambda^{*} \ll 1$ as

$$
G\left(x ; x_{0}, \lambda^{*}\right)=-\frac{1}{|\Omega| \lambda^{*}}+G_{m}\left(x ; x_{0}\right)+O\left(\lambda^{*}\right)=-\frac{1}{|\Omega| \lambda^{*}}-\frac{1}{2 \pi} \log \left|x-x_{0}\right|+R_{m}\left(x ; x_{0}\right)+O\left(\lambda^{*}\right) .
$$

Comparing terms in (2.12) and $(2.7 b)$, we obtain

$$
R\left(x ; x_{0}, \lambda^{*}\right)=-\frac{1}{|\Omega| \lambda^{*}}+R_{m}\left(x ; x_{0}\right)+O\left(\lambda^{*}\right) .
$$

Substituting this expression into (2.9), we get the following two-term asymptotic result:

Proposition 2.1:(One Hole) For $\varepsilon \rightarrow 0$, the first eigenvalue $\lambda_{0}$ of (1.1) has the two-term asymptotic behaviour

$$
\lambda_{0}(\varepsilon)=\frac{2 \pi v}{|\Omega|\left(1+2 \pi v R_{m}\left(x_{0} ; x_{0}\right)\right)}+O\left(v^{3}\right)
$$

Here $v=-1 / \log (\varepsilon d)$, and $d$ is determined from the inner problem (2.2). An infinite-order logarithmic expansion for $\lambda_{0}$ is given by $\lambda_{0} \sim \lambda^{*}$, where $\lambda^{*}$ is the root of (2.9).

\subsection{Multiple holes}

Now, we extend the development above to the case of $N$ holes. Much of the analysis above remains the same, except now the single hole $x_{0}$ is replaced with $x_{i}$, for $i=1, \ldots, N$. 
The equivalent formulation for $N$ holes becomes

$$
\begin{gathered}
\Delta u^{*}+\lambda^{*} u^{*}=0, \quad x \in \Omega \backslash \cup_{i=1}^{N}\left\{x_{i}\right\} ; \quad \int_{\Omega}\left(u^{*}\right)^{2} d x=1 ; \quad \partial_{n} u^{*}=0, \quad x \in \partial \Omega, \\
u^{*} \sim A_{i} v_{i} \log \left|x-x_{i}\right|+A_{i}, \quad x \rightarrow x_{i}, \quad i=1, \ldots, N
\end{gathered}
$$

Here, $v_{i}=-1 / \log \left(\varepsilon d_{i}\right)$, where $d_{i}$ is a shape-dependent parameter for the $i$ th hole. Also, we have $N$ unknowns, $A_{i}$, for $i=1, \ldots, N$, with one normalization condition for $u^{*}$. This condition effectively sets one relation between the $A_{i}$, for $i=1, \ldots, N$.

We write $u^{*}$ in terms of the Helmholtz Green's function defined in (2.7), and then take the limit $x \rightarrow x_{i}$ to get

$$
u^{*}=-2 \pi \sum_{k=1}^{N} A_{k} v_{k} G\left(x ; x_{k}, \lambda^{*}\right) \sim A_{i} v_{i}\left(\log \left|x-x_{i}\right|-2 \pi v_{i} R\left(x_{i} ; x_{i}, \lambda^{*}\right)\right)-2 \pi \sum_{\substack{k=1 \\ k \neq i}}^{N} A_{k} v_{k} G\left(x_{i} ; x_{k}, \lambda^{*}\right) .
$$

The matching condition is that the expressions in (2.15b) and (2.16) agree. The logarithmic terms agree, and from the remaining terms, we obtain an $N \times N$ homogeneous linear system to solve for the $A_{i}$

$$
A_{i}\left(1+2 \pi v_{i} R\left(x_{i} ; x_{i}, \lambda^{*}\right)\right)+2 \pi \sum_{\substack{k=1 \\ k \neq i}}^{N} A_{k} v_{k} G\left(x_{i} ; x_{k}, \lambda^{*}\right)=0, \quad i=1, \ldots, N
$$

A solution exists to (2.17) when the following determinant is zero:

$$
\left|\begin{array}{cccc}
\left(1+2 \pi v_{1} R_{11}\left(\lambda^{*}\right)\right) & 2 \pi v_{2} G_{12}\left(\lambda^{*}\right) & \cdots & 2 \pi v_{N} G_{1 N}\left(\lambda^{*}\right) \\
2 \pi v_{1} G_{21}\left(\lambda^{*}\right) & \left(1+2 \pi v_{2} R_{22}\left(\lambda^{*}\right)\right) & & \vdots \\
\vdots & \ldots & \ddots & \\
2 \pi v_{1} G_{N 1}\left(\lambda^{*}\right) & \cdots & 2 \pi v_{N-1} G_{N(N-1)}\left(\lambda^{*}\right) & \left(1+2 \pi v_{N} R_{N N}\left(\lambda^{*}\right)\right)
\end{array}\right|=0 .
$$

Here we have defined $R_{i i}\left(\lambda^{*}\right)=R\left(x_{i} ; x_{i}, \lambda^{*}\right), G_{i k}\left(\lambda^{*}\right)=G\left(x_{i} ; x_{k}, \lambda^{*}\right)$, for $i \neq k$, and $v_{i}=$ $-1 / \log \left(\varepsilon d_{i}\right)$ for $i=1, \ldots, N$. We need the solution $\lambda^{*}\left(v_{1}, \ldots, v_{N}\right)$ of $(2.18)$ that tends to zero as $v_{i} \rightarrow 0$. Equation (2.17) provides an expression for $\lambda^{*}(v)$ that sums all the logarithmic terms in the asymptotic expansion of $\lambda_{0}(\varepsilon)$.

As with the case for one hole in the domain, we derive an asymptotic formula for $\lambda^{*}$ that has an error of $O\left(v^{3}\right)$. This formula is again determined in terms of the Neumann Green's function $G_{m}$ and its regular part $R_{m}$, defined in (1.5). By using (2.12) and (2.13) in (2.18), we obtain a homogeneous linear system for the $A_{i}$

$$
A_{i}\left[1+2 \pi v_{i} R_{m}\left(x_{i} ; x_{i}\right)-\frac{2 \pi v_{i}}{|\Omega| \lambda^{*}}\right]+2 \pi \sum_{\substack{j=1 \\ j \neq i}}^{N} A_{j} v_{j}\left[-\frac{1}{|\Omega| \lambda^{*}}+G_{m}\left(x_{i} ; x_{j}\right)\right]=0, \quad i=1, \ldots, N .
$$


It is convenient to write (2.19) in matrix form as

$$
\begin{aligned}
& \mathscr{C} \boldsymbol{a}=\frac{2 \pi}{|\Omega| \lambda^{*}} \mathscr{B} \mathscr{V} \boldsymbol{a} ; \quad \mathscr{V} \equiv\left(\begin{array}{cccc}
v_{1} & 0 & \cdots & 0 \\
0 & \ddots & \cdots & 0 \\
\vdots & \vdots & \ddots & \vdots \\
0 & 0 & \cdots & v_{N}
\end{array}\right), \\
& \mathscr{B} \equiv\left(\begin{array}{cccc}
1 & 1 & \cdots & 1 \\
1 & \ddots & \cdots & 1 \\
\vdots & \vdots & \ddots & \vdots \\
1 & 1 & \cdots & 1
\end{array}\right), \quad \boldsymbol{a} \equiv\left(\begin{array}{c}
A_{1} \\
\vdots \\
A_{N}
\end{array}\right) .
\end{aligned}
$$

In $(2.20 a)$, the matrix $\mathscr{C}$ is defined in terms of the Green's function matrix $\mathscr{G}$ by

$$
\mathscr{C}=I+2 \pi \mathscr{G} \mathscr{V}
$$

where

$$
\mathscr{G} \equiv\left(\begin{array}{ccccc}
R_{m}\left(x_{1} ; x_{1}\right) & G_{m}\left(x_{1} ; x_{2}\right) & \ldots & \ldots & G_{m}\left(x_{1} ; x_{N}\right) \\
G_{m}\left(x_{2} ; x_{1}\right) & R_{m}\left(x_{2} ; x_{2}\right) & G_{m}\left(x_{2} ; x_{3}\right) & \ldots & \ldots \\
\vdots & \vdots & \ddots & \vdots & \vdots \\
\ldots & \ldots & G_{m}\left(x_{N-1} ; x_{N-2}\right) & R_{m}\left(x_{N-1} ; x_{N-1}\right) & G_{m}\left(x_{N-1} ; x_{N}\right) \\
G_{m}\left(x_{N} ; x_{1}\right) & \ldots & \ldots & G_{m}\left(x_{N} ; x_{N-1}\right) & R_{m}\left(x_{N} ; x_{N}\right)
\end{array}\right) .
$$

Let $v_{m}=\max _{j=1, \ldots, N} v_{j}$. Then, for $v_{m}$ sufficiently small, we can invert $\mathscr{C}$, to obtain that $\lambda^{*}$ is an eigenvalue of the matrix eigenvalue problem

$$
\mathscr{A} \boldsymbol{a}=\lambda^{*} \boldsymbol{a}, \quad \mathscr{A}=\frac{2 \pi}{|\Omega|} \mathscr{C}^{-1} \mathscr{B} \mathscr{V} .
$$

By using this representation of $\lambda^{*}$ we obtain the following result:

Proposition 2.2:( $N$ Holes) For $\varepsilon \rightarrow 0$, the first eigenvalue $\lambda_{0}$ of (1.1) has the two-term asymptotic behaviour

$$
\lambda_{0}(\varepsilon) \sim \lambda^{*}, \quad \lambda^{*}=\frac{2 \pi}{|\Omega|}\left(\sum_{j=1}^{N} v_{j}-2 \pi \sum_{j=1}^{N} \sum_{k=1}^{N} v_{j} v_{k}(\mathscr{G})_{j k}\right)+O\left(v_{m}^{3}\right)
$$

Here $(\mathscr{G})_{j k}$ are the entries of the matrix $\mathscr{G}$ defined in $(2.20 \mathrm{c})$.

Proof: We first notice that the matrix $\mathscr{B} \mathscr{V}$ has rank one, since $\mathscr{V}$ is diagonal and $\mathscr{B}=\boldsymbol{e}_{0} \boldsymbol{e}_{0}^{t}$, where $\boldsymbol{e}_{0}^{t}=(1,1, \ldots, 1)$. This implies that $\mathscr{A}$ has rank one, and so $\lambda^{*}$ is the unique nonzero eigenvalue of $\mathscr{A}$. Hence, $\lambda^{*}=$ Trace $\mathscr{A}$. By using the structure of $\mathscr{A}$ in $(2.21)$, we obtain 
from a simple calculation that

$$
\lambda^{*}=\frac{2 \pi}{|\Omega|} \sum_{j=1}^{N} v_{j}\left(\sum_{k=1}^{N} c_{j k}\right), \quad c_{j k} \equiv\left(\mathscr{C}^{-1}\right)_{j k} .
$$

Finally, we use the asymptotic inverse $\mathscr{C}^{-1} \sim I-2 \pi \mathscr{G} \mathscr{V}+\cdots$ for $v_{m} \ll 1$ to calculate $c_{j k}$. Substituting this result into (2.23) we obtain (2.22).

As a Corollary to Proposition 2.2, we obtain the following result for $N$ identical holes:

Corollary 2.3:( $N$ Identical Holes) Suppose that the $N$ holes are identical, in the sense that $\widetilde{\varepsilon} \equiv \varepsilon d_{j}$ is independent of $j$. Then, (2.22) can be written as the two-term expansion

$$
\lambda_{0}(\varepsilon) \sim \lambda^{*}, \quad \lambda^{*}=\frac{2 \pi N v}{|\Omega|}-\frac{4 \pi^{2} v^{2}}{|\Omega|} p\left(x_{1}, \ldots, x_{N}\right)+O\left(v^{3}\right),
$$

where $v \equiv-1 / \log \tilde{\varepsilon}$, and the function $p\left(x_{1}, \ldots, x_{N}\right)$ is defined by

$$
p\left(x_{1}, \ldots, x_{N}\right)=\sum_{j=1}^{N} \sum_{k=1}^{N}(\mathscr{G})_{j k} \equiv N \boldsymbol{e}^{t} \mathscr{G}_{\boldsymbol{e}}=\sum_{k=1}^{N}\left(R_{m}\left(x_{k} ; x_{k}\right)+\sum_{\substack{j=1 \\ j \neq k}}^{N} G_{m}\left(x_{j} ; x_{k}\right)\right) .
$$

Here $(\mathscr{G})_{j k}$ are the entries in the matrix $\mathscr{G}$ in $(2.20 \mathrm{c})$, and $\boldsymbol{e}$ is the unit vector $\boldsymbol{e}=$ $N^{-1 / 2}(1, \ldots, 1)^{t}$. For $N$ circular holes of radius $\varepsilon$, then $d_{j}=1$ for $j=1, \ldots, N$, and so $\tilde{\varepsilon} \equiv \varepsilon$.

For $v \ll 1$, we observe from (2.24) for $N$ identical holes that the eigenvalue $\lambda_{0}(\varepsilon)$ is largest when the hole locations $x_{1}, \ldots, x_{N}$ are chosen to minimize $p\left(x_{1}, \ldots, x_{N}\right)$. Since $\mathscr{G}$ is a symmetric matrix, it can be diagonalized as $\mathscr{G}=\mathscr{S} \Lambda \mathscr{S}^{t}$, where $\mathscr{S}$ is an orthogonal matrix of eigenvectors $s_{j}$ for $j=1, \ldots, N$, and $\Lambda$ is the diagonal matrix of eigenvalues $\mu_{j}$ for $j=1, \ldots, N$. Then, since $s_{j}$ and $e$ are unit vectors, we can write $p$ in (2.25) as

$$
p\left(x_{1}, \ldots, x_{N}\right)=N \sum_{j=1}^{N} \mu_{j} \cos ^{2} \phi_{j}, \quad \text { where } \quad \cos \phi_{j} \equiv \boldsymbol{s}_{j}^{t} \boldsymbol{e}, \quad \sum_{j=1}^{N} \cos ^{2} \phi_{j}=1 .
$$

Here $\phi_{j}$ represents the angle between $\boldsymbol{s}_{j}$ and $\boldsymbol{e}$. Since $\mathscr{S}$ is an orthogonal matrix and $\boldsymbol{e}$ is a unit vector, the vector $\mathscr{S}^{t} \boldsymbol{e}$ has length one. Hence, the spectral representation of our optimization problem is to minimize $p$ in (2.26) subject to the constraint $\sum_{j=1}^{N} \cos ^{2} \phi_{j}=1$.

\section{One hole in a simply connected domain}

In this section we consider (1.1) for one hole centred at some point $x_{0} \in \Omega$. We assume that $\Omega$ is a simply-connected domain that can be non-convex. From (2.14) of Proposition 2.1, the fundamental eigenvalue satisfies

$$
\lambda_{0}(\varepsilon) \sim \frac{2 \pi v}{|\Omega|}-\frac{4 \pi^{2} v^{2}}{|\Omega|} R_{m}\left(x_{0} ; x_{0}\right)+O\left(v^{3}\right) .
$$

Therefore, $\lambda_{0}$ is maximized when the hole is centred at a point that minimizes $R_{m}\left(x_{0} ; x_{0}\right)$. An interesting question is to determine whether there is a unique point $x_{0}$ in $\Omega$ that 
minimizes $R_{m}\left(x_{0} ; x_{0}\right)$, and consequently maximizes $\lambda_{0}$. To study this question, we require a formula for $\left.\nabla R_{m}\left(x ; x_{0}\right)\right|_{x=x_{0}}$. By using a complex-variable method, such a formula was derived in [15] in the context of studying equilibrium spike locations for the GiererMeinhardt reaction-diffusion system. That result, which pertains to certain classes of mappings of the unit disk, is as follows:

Proposition 3.1([Theorem 4.1 of 15]) Let $f(z)$ be a complex mapping of the unit disk $B$ onto $\Omega$, which satisfies the following conditions:

(i) $f$ is analytic and is invertible on $\bar{B}$. Here $\bar{B}$ is $B$ together with its boundary $\partial B$.

(ii) $f$ has only simple poles at the points $z_{1}, z_{2}, \ldots, z_{k}$, and $f$ is bounded at infinity.

(iii) $f=g / h$ where both $g$ and $h$ are analytic on the entire complex plane, with $g\left(z_{i}\right) \neq 0$.

(iv) $\overline{f(z)}=f(\bar{z})$.

On the image domain $\Omega=f(B)$, let $G_{m}$ and $R_{m}$ be the Neumann Green's function and its regular part, respectively, defined in (1.5). Let $R_{m 0}$ and $\nabla R_{m 0}$ be the value of $R_{m}$ and its gradient evaluated at $x_{0}$. Then,

$$
\nabla R_{m 0}=\frac{\nabla s\left(z_{0}\right)}{\overline{f^{\prime}\left(z_{0}\right)}}
$$

where $z_{0} \in B$ satisfies $x_{0}=f\left(z_{0}\right)$, with $x_{0} \in \Omega$, and $\nabla s\left(z_{0}\right)$ is given by

$\nabla s\left(z_{0}\right)=\frac{1}{2 \pi}\left(\frac{z_{0}}{1-\left|z_{0}\right|^{2}}+\frac{f^{\prime \prime}\left(\bar{z}_{0}\right)}{2 f^{\prime}\left(\bar{z}_{0}\right)}\right)+\frac{f^{\prime}\left(\bar{z}_{0}\right)\left(f\left(z_{0}\right)-f\left(\frac{1}{\bar{z}_{0}}\right)\right)}{2|\Omega|}+\frac{\sum_{j=1}^{k} \tau_{j}\left(\frac{1}{z_{j}-\bar{z}_{0}}+\frac{z_{j}}{1-z_{j} \bar{z}_{0}}\right)}{2|\Omega|}$.

Here $|\Omega|$, denoting the area of $\Omega$, and $\tau_{j}$ are defined by

$$
\tau_{j} \equiv \frac{g\left(z_{j}\right) f^{\prime}\left(\frac{1}{z_{j}}\right)}{z_{j}^{2} h^{\prime}\left(z_{j}\right)}, \quad \Omega=-\pi \sum_{j=1}^{k} \tau_{j} .
$$

In the equation above, and for the rest of this section, we will treat vectors $v=\left(v_{1}, v_{2}\right)$ as complex numbers $v_{1}+i v_{2}$. Therefore, $v w$ is assumed to be complex multiplication.

For our first example we take the following class of mappings considered in $[11,15]$ :

$$
f(z)=\frac{\left(1-b^{2}\right) z}{z^{2}-b^{2}} .
$$

Here $b$ is real and $b>1$. The resulting domain $\Omega=f(B)$ is shown in Fig. 1(a) for several values of $b$. Notice that $\Omega \rightarrow B$ as $b \rightarrow \infty$. In the other limit where $\varepsilon \equiv b-1 \rightarrow 0^{+}, \Omega$ approaches the union of two circles centred at $\left( \pm \frac{1}{2}, 0\right)$, with radius $\frac{1}{2}$, which are connected by a narrow channel of length $2 \varepsilon+O\left(\varepsilon^{2}\right)$. It is easy to see that $\Omega$ is non-convex only when $1<b<1+\sqrt{2}$. For this example, it was shown using (3.3) (see $\S 4.1$ of [15]) that there is a unique root to $\nabla R_{m 0}=0$ for any $b>1$, and that this root is located at $x_{0}=0$. To show this, the vector field generated by (3.3) was plotted in Fig. 3 of [15] for points in the first quadrant. For points along the real axis where $z_{0}=\bar{z}_{0}=\xi$, and $-1<\xi<1$, a 

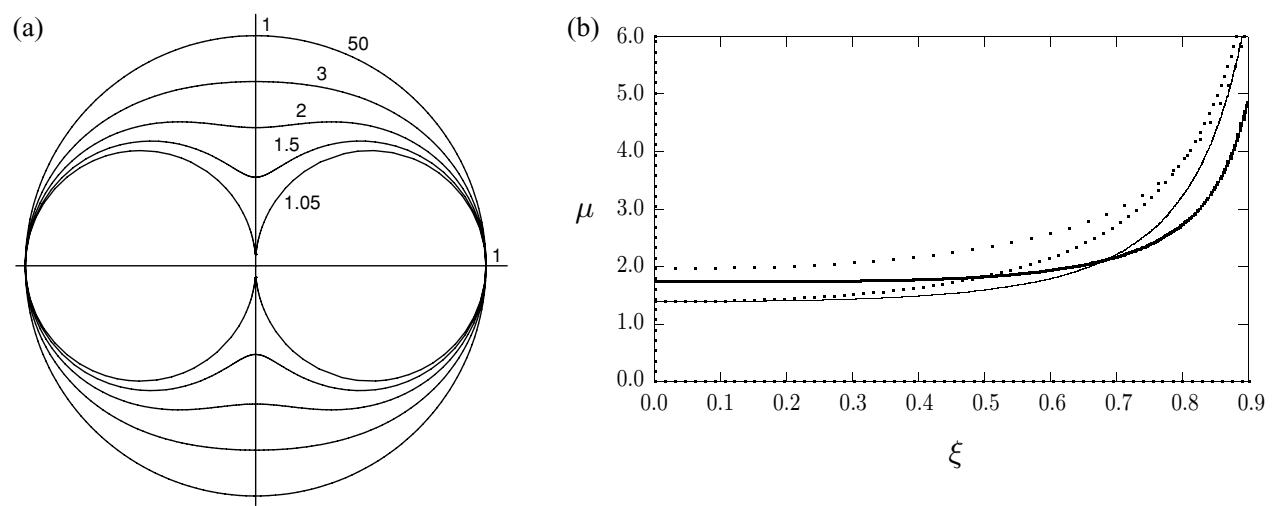

FIGURE 1. (a): The boundary of $\Omega=f(B)$, with $f(z)$ as given in (3.4), for the values of $b$ as shown. (b): Plots of $\mu(\xi)$, defined in (3.5), on $0 \leqslant \xi<1$ for $b=1.05$ (heavy solid curve), $b=1.2$ (solid curve), $b=1.5$ (dotted curve), and $b=5.0$ (widely spaced dots).

straightforward calculation from (3.3) yields that $\nabla s\left(z_{0}\right)=\frac{\xi \mu(\xi)}{2 \pi}$, where the even function $\mu(\xi)$ is defined by

$$
\mu(\xi) \equiv \frac{2 b^{2}\left(b^{2}+1\right)-\left(\xi^{2}+b^{2}\right)^{2}}{\left(b^{4}-\xi^{4}\right)\left(1-\xi^{2}\right)}+\frac{1}{b^{2} \xi^{2}-1}\left[b^{2}+\frac{\left(b^{4}-1\right)^{2}\left(b^{2}+1\right)\left(\xi^{2}+b^{2}\right)\left(\xi^{2}-1\right)}{\left(b^{4}+1\right)\left(b^{2}-\xi^{2}\right)^{3}}\right] .
$$

Since $f(0)=0$, the uniqueness of the root to $\nabla R_{m 0}=0$ follows provided that $\mu(\xi)$ is of one sign for $0 \leqslant \xi<1$ and for any $b>1$. It is easy to show that $\mu(0)>0$ for any $b>1$, $\mu \rightarrow+\infty$ as $\xi \rightarrow 1^{-}$, and $\mu^{\prime}(\xi)>0$ on $0<\xi<1$. Therefore $\mu(\xi)>0$ on $0<\xi<1$, and the uniqueness of the root to $\nabla R_{m 0}=0$ follows. In Fig. $1(b)$ we plot $\mu(\xi)$ on $0<\xi<1$ for several values of $b$. Since $R_{m 0} \rightarrow+\infty$ as $x_{0}$ approaches a point on the boundary of $\Omega$ from within (cf. Appendix A of [15]), we conclude that $x_{0}$ is the unique minimum point of $R_{m}\left(x_{0} ; x_{0}\right)$. Therefore, from (3.1), $x_{0}=0$ is the unique point in $\Omega$ that maximizes $\lambda_{0}$.

The interpretation of this result, within the context of the Brownian motion problem of $\S 1$, is that the best place to catch a Brownian particle in a symmetric dumbbell-shaped domain is to locate the trap in the centre of the channel connecting the two lobes of the dumbbell. Such a trap location minimizes the expected lifetime of the Brownian particle and is preferable to locating the trap in the centre of one of the two dumbbell lobes.

In [15] a boundary integral method was used to show the uniqueness of the root to $\nabla R_{m 0}=0$ for a few other non-convex domains (see Fig. 7 and Fig. 8 of [15]). Based on this additional numerical evidence, it was conjectured in [15] that there is always a unique root to $\nabla R_{m 0}=0$ in any simply-connected non-convex domain. Our next example, based on an asymmetric dumbbell-shaped domain, shows that this conjecture is, in general, false.

Let $B$ be the unit ball, and consider the mapping $\Omega=f(B)$ generated by

$$
f(z)=-\frac{\kappa z}{(z-a)(z+b)}, \quad a>1, \quad b>1, \quad \kappa \equiv(a-1)(b+1) .
$$


(1)

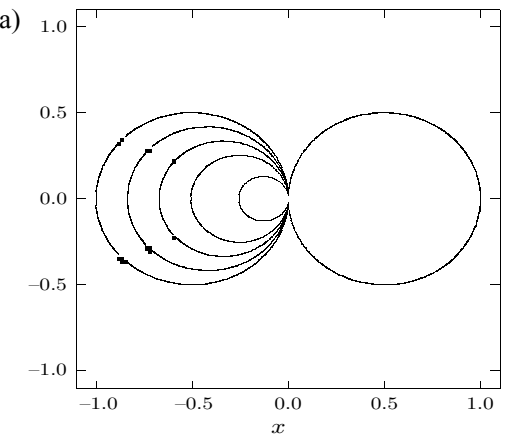

(b)

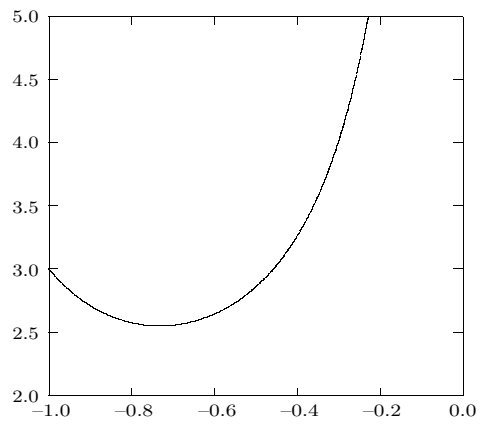

Figure 2. (a): The boundary of $\Omega=f(B)$ for (3.6), where $a=1+\varepsilon, b=1+\varepsilon \gamma$, and $\varepsilon=0.02$. The nested 'circles' in the left lobe of the dumbbell are for $\gamma=1.0,1.2,1.5,2.0,4.0$. The radii of these circles decrease as $\gamma$ increases. (b): Plot of $\gamma^{2}=H\left(y_{0}\right)$, versus $y_{0}$, where $H\left(y_{0}\right)$ is given in (3.18). This plot determines the zeroes of $\nabla R_{m 0}=0$ inside the right lobe of the dumbbell.

Here $\kappa$ is chosen so that $f(1)=1$. For this example, a lengthy but straightforward calculation using (3.3) yields

$$
\nabla s\left(z_{0}\right)=\frac{1}{2 \pi}\left[\frac{z_{0}}{1-\left|z_{0}\right|^{2}}+\frac{a b^{2}+3 a b \bar{z}_{0}+\bar{z}_{0}^{3}-b a^{2}}{\left(a-\bar{z}_{0}\right)\left(b+\bar{z}_{0}\right)\left(\bar{z}_{0}^{2}+a b\right)}\right]+\frac{T_{2}}{2|\Omega|}+\frac{T_{3}}{2|\Omega|}
$$

where $T_{2}$ and $T_{3}$ are given by

$$
\begin{aligned}
T_{2}= & \frac{\kappa^{2}\left(\bar{z}_{0}^{2}+a b\right)\left(\left|z_{0}\right|^{2}-1\right)\left(z_{0}+a b \bar{z}_{0}\right)}{\left(\bar{z}_{0}-a\right)^{2}\left(\bar{z}_{0}+b\right)^{2}\left(a-z_{0}\right)\left(b+z_{0}\right)\left(1+b \bar{z}_{0}\right)\left(-1+a \bar{z}_{0}\right)}, \\
T_{3}= & \frac{\kappa^{2} a\left(1+a^{3} b\right)\left(1-2 a \bar{z}_{0}+a^{2}\right)}{(a+b)(a-1)^{2}(a+1)^{2}(1+a b)^{2}\left(a-\bar{z}_{0}\right)\left(-1+a \bar{z}_{0}\right)} \\
& +\frac{\kappa^{2} b\left(1+a b^{3}\right)\left(1+2 b \bar{z}_{0}+b^{2}\right)}{(a+b)(b-1)^{2}(b+1)^{2}(1+a b)^{2}\left(b+\bar{z}_{0}\right)\left(1+b \bar{z}_{0}\right)} .
\end{aligned}
$$

The area of the domain is given by

$$
|\Omega|=\kappa^{2} \pi\left[\frac{a\left(1+a^{3} b\right)\left(b^{2}-1\right)^{2}+b\left(a^{2}-1\right)^{2}\left(1+a b^{3}\right)}{(a+b)(1+a b)^{2}\left(a^{2}-1\right)^{2}\left(b^{2}-1\right)^{2}}\right] .
$$

Let $\gamma>1$ and $\varepsilon \ll 1$, and define $a$ and $b$ by

$$
a=1+\varepsilon, \quad b=1+\varepsilon \gamma .
$$

In the limit $\varepsilon \rightarrow 0$, it is easy to see that $\Omega=f(B)$ approaches the union of two circles; a larger circle centred at $(1 / 2,0)$ of radius $1 / 2$, and a smaller circle centred at $(-1 /(2 \gamma), 0)$ of radius $1 /(2 \gamma)$. Plots of $\Omega$ are shown in Fig. $2(a)$ for $\varepsilon=0.02$, and for several values of 
$\gamma$. For $\varepsilon \rightarrow 0^{+}$, we have from (3.6) and $(3.7 d)$ that

$$
\kappa \sim 2 \varepsilon, \quad|\Omega| \sim \frac{\pi\left(1+\gamma^{2}\right)}{4 \gamma^{2}} .
$$

We now look for roots of $\nabla R_{m 0}=0$ where $x_{0}=f\left(z_{0}\right)$ and $z_{0} \in \mathscr{B}$. It is clear from (3.6) and (3.8) that there are two distinguished limits: $\left|z_{0}\right|<1$ and $1-\left|z_{0}\right|=O(\varepsilon)$ for which $x_{0}=O(\varepsilon)$ and $x_{0}=O(1)$, respectively.

Assume first that $1-\left|z_{0}\right|=O(1)$. We substitute (3.8) into (3.7) to obtain $T_{2}=O\left(\varepsilon^{2}\right) \ll 1$, and that

$$
\nabla s\left(z_{0}\right) \sim \frac{1}{2 \pi}\left[\frac{z_{0}}{1-\left|z_{0}\right|^{2}}+\frac{3 \bar{z}_{0}+\bar{z}_{0}^{3}}{\left(1-\bar{z}_{0}^{2}\right)\left(\bar{z}_{0}^{2}+1\right)}\right]+\frac{\kappa^{2}}{16|\Omega| \varepsilon^{2}}\left(\frac{1}{\bar{z}_{0}-1}+\frac{1}{\gamma^{2}\left(\bar{z}_{0}+1\right)}\right) .
$$

Substituting (3.9) into (3.10), we obtain after a little algebra that

$$
\nabla s\left(z_{0}\right) \sim \frac{1}{2 \pi}\left[\frac{2 \operatorname{Re}\left(z_{0}\right)}{\left(1-\left|z_{0}\right|^{2}\right)\left(1+\bar{z}_{0}^{2}\right)}+\frac{2 \beta}{\bar{z}_{0}^{2}-1}\right]
$$

where $\beta \equiv\left(\gamma^{2}-1\right) /\left(\gamma^{2}+1\right)$. Setting $\nabla s\left(z_{0}\right)=0$, we get

$$
\frac{\operatorname{Re}\left(z_{0}\right)}{1-\left|\bar{z}_{0}\right|^{2}}=\beta\left(\frac{1+\bar{z}_{0}^{2}}{1-\bar{z}_{0}^{2}}\right)=\frac{\beta}{\left|1-z_{0}^{2}\right|^{2}}\left(1-\left|\bar{z}_{0}\right|^{4}-4 i \operatorname{Re}\left(z_{0}\right) \operatorname{Im}\left(z_{0}\right)\right) \text {. }
$$

By taking imaginary parts of (3.12) we get $\operatorname{Im}\left(z_{0}\right)=0$. Then, upon setting $z_{0}=\xi$, where $|\xi|<1$ is real, we obtain

$$
\xi^{2}-\frac{\xi}{\beta}+1=0, \quad \beta \equiv \frac{\gamma^{2}-1}{\gamma^{2}+1} .
$$

Let $\gamma>1$. Then, for $0<\beta<1 / 2$, there is a unique root $\xi_{0}$ to (3.13) inside the unit disk. Notice that $\xi_{0} \rightarrow 1^{-}$as $\beta \rightarrow(1 / 2)^{-}$. The root $x_{0}=f\left(\xi_{0}\right)$ to $\nabla R_{m 0}=0$ in $\Omega$ is obtained by letting $\varepsilon \rightarrow 0$ in (3.6) and (3.8). In this way, we obtain for $1<\gamma<\sqrt{3}$ that there is a root to $\nabla R_{m 0}=0$ in $\Omega$ at the point $x_{0}=f\left(\xi_{0}\right)$, where

$$
x_{0}=\frac{2 \varepsilon \beta}{\sqrt{1-4 \beta^{2}}}=\frac{2 \varepsilon\left(\gamma^{2}-1\right)}{\sqrt{10 \gamma^{2}-3\left(\gamma^{4}+1\right)}}, \quad \xi_{0}=\frac{1}{2 \beta}-\sqrt{\frac{1}{(2 \beta)^{2}}-1}
$$

For $\gamma \geqslant 1$, we have from (3.13) that $0 \leqslant \beta<1$. However, from (3.14) we observe that $\xi_{0} \sim 1$ when $\beta \sim \frac{1}{2}+O\left(\varepsilon^{2}\right)$. Therefore, the analysis leading to (3.14), which assumed that $1-\left|z_{0}\right| \gg O(\varepsilon)$, becomes invalid when $\beta \sim \frac{1}{2}$. To determine if there are any roots of $\nabla R_{m 0}=0$ when $\frac{1}{2}<\beta<1$, or equivalently when $\gamma>\sqrt{3}$, we must study the distinguished limit where $1-\left|z_{0}\right|=O(\varepsilon)$ in (3.7). For this case, we look for a root of $\nabla R_{m 0}=0$ with $z_{0}$ real and

$$
a=1+\varepsilon, \quad b=1+\gamma \varepsilon, \quad z_{0}=1-\varepsilon \mu .
$$


Substituting (3.15) and (3.9) into (3.7), and letting $\varepsilon \rightarrow 0$, we obtain that

$$
\begin{aligned}
\nabla s\left(z_{0}\right) & \sim \frac{1}{2 \pi \varepsilon}\left[\frac{1}{2 \mu}+\frac{1}{\mu+1}\right]+\frac{\mu}{4 \varepsilon|\Omega|\left(1-\mu^{2}\right)}\left[1-\frac{4}{(1+\mu)^{2}}\right]+O(1), \\
& \sim \frac{1}{4 \pi \varepsilon(\mu+1)}\left[\frac{(3 \mu+1)}{\mu}-\frac{4 \mu}{\left(\gamma^{-2}+1\right)} \frac{(\mu+3)}{(1+\mu)^{2}}\right]+O(1) .
\end{aligned}
$$

Setting $\nabla s\left(z_{0}\right)=0$, we obtain a relation between $\gamma$ and $\mu$ of the form

$$
\gamma^{2}=\frac{(3 \mu+1)(\mu+1)^{2}}{(\mu-1)\left(\mu^{2}+6 \mu+1\right)}
$$

Substituting (3.15) into the mapping (3.6), we obtain a relation between $x_{0}$ and $\mu$. Combining this relation with (3.17), and defining $y_{0}$ by $y_{0}=2 x_{0}-1$, we get

$$
x_{0}=\frac{1}{1+\mu}, \quad y_{0}=2 x_{0}-1, \quad \gamma^{2}=H\left(y_{0}\right) \equiv \frac{2-y_{0}}{y_{0}\left(y_{0}^{2}-2\right)} .
$$

In Fig. $2(b)$ we plot $\gamma^{2}$ versus $y_{0}$ on $-1<y_{0}<0$, which corresponds to the interval $0<x_{0}<1 / 2$. A simple calculation shows that $H\left(y_{0}\right)>0$ for $-1<y_{0}<0$. On this range, $H(y)$ has a unique minimum at $y=y_{0 c}$, with $H^{\prime}(y)<0$ for $-1<y_{0}<y_{0 c}$ and $H^{\prime}(y)>0$ for $y_{0 c}<y_{0}<0$, where

$$
y_{0 c}=1-\sqrt{3}, \quad x_{0 c}=1-\frac{\sqrt{3}}{2}, \quad \gamma_{c}=\sqrt{H\left(y_{0 c}\right)}=\sqrt{\frac{5+\sqrt{27}}{4}}=1.59657 .
$$

We summarize our result for the asymmetric dumbbell-shaped domain as follows:

Proposition 3.2 Consider the mapping (3.6) of the unit disk, with $a=1+\varepsilon$ and $b=1+\varepsilon \gamma$, where $\varepsilon>0$ and $\gamma>1$. Suppose that $1<\gamma<\gamma_{c}=1.59657$. Then, for $\varepsilon \ll 1$, there is a unique root to $\nabla R_{m 0}=0$ in $\Omega$ at the point $x_{0}=f\left(\xi_{0}\right)=O(\varepsilon)$, where $x_{0}$ is given in (3.14). On the range $1.59657<\gamma<\sqrt{3}, \nabla R_{m 0}=0$ has three roots. The smallest root is $x_{0}=O(\varepsilon)$, as given by (3.14), and the other two roots $x_{0 L}$ and $x_{0 R}$, which satisfy $x_{0 L}<1-\sqrt{3} / 2$ and $x_{0 R}>1-\sqrt{3} / 2$, are the solutions of $\gamma^{2}=H\left(2 x_{0}-1\right)$, where $H\left(y_{0}\right)$ is given in (3.18). As $\gamma \rightarrow \sqrt{3}$ from below, the root $x_{0 L}$ tends to zero and annihilates the smallest root $x_{0}$ in a saddle-node bifurcation. For $\gamma>\sqrt{3}, \nabla R_{m 0}=0$ has a unique root $x_{0 R}$, which satisfies $\gamma^{2}=H\left(2 x_{0}-1\right)$. As $\gamma \rightarrow \infty$, we have that $x_{0 R} \rightarrow 1 / 2$.

This result shows that for a slightly asymmetric dumbbell-shaped domain, where $1<\gamma<1.59657$, the optimum place to maximize $\lambda_{0}$ in $(3.1)$ is to put the trap in the channel region of the dumbbell, but shifted slightly towards the side of the largest lobe. For $\gamma \gg 1$, where the left lobe of the dumbbell is very small (see Fig. 2(a)) the optimum place to insert the trap is near the centre of the right lobe of the dumbbell. These two limiting results are certainly plausible in the context of the Brownian motion problem of $\S 1$. However, the results of Proposition 3.2 show that the transition between these two regimes has a complicated bifurcation structure for $1.59657<\gamma<\sqrt{3}$, where $\lambda_{0}$ has two local maxima and one local minimum. 

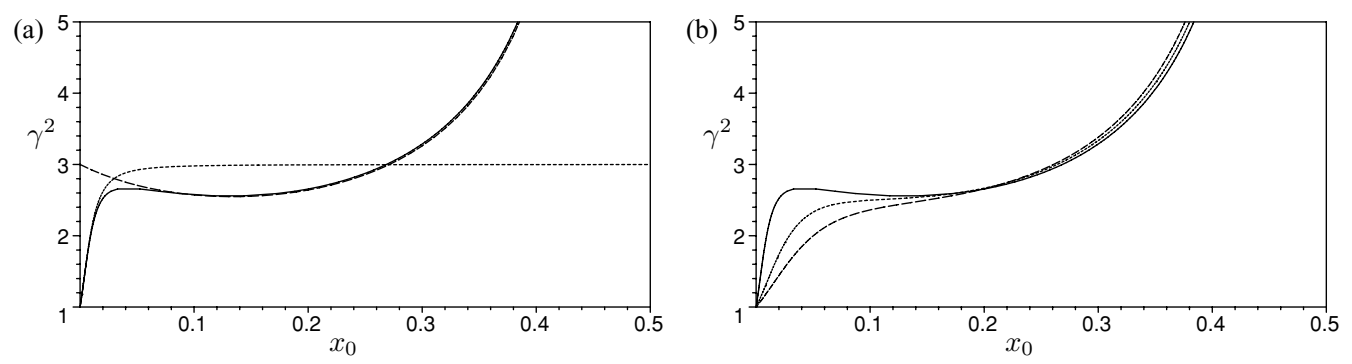

Figure 3. (a): The ratio of areas $\gamma^{2}$ as a function of the roots $x_{0}$ of $\nabla R_{m}=0$, with $\varepsilon=0.01$. The solid curve is obtained by solving for the roots in the exact formula (3.7), (3.8). The dotted and dashed curves represent the reduced asymptotic approximations (3.14) and (3.18), respectively. (b): Plots of the roots $x_{0}$ versus $\gamma^{2}$ for three different values of $\varepsilon$. The solid, dotted, and dashed curves correspond to $\varepsilon=0.01,0.03$, and 0.05 , respectively.

To illustrate Proposition 3.2, in Fig. 3(a) we plot the locations $x_{0}$ of the roots of $\nabla R_{m 0}=0$ versus $\gamma^{2}$ for $\gamma^{2}>1$ and $x_{0}>0$. In this figure, we compare the asymptotic formulae (3.14) and (3.18) with the exact solution, obtained by numerically solving for the roots of (3.7), (3.8) with $\varepsilon=0.01$. As predicted by the theory, there are multiple roots in the limit $\varepsilon \rightarrow 0$ whenever $\gamma^{2} \in((5+\sqrt{24}) / 4,3)$. In Fig. 3(b), we plot the roots for several values of $\varepsilon$. Notice that as $\varepsilon$ is increased, the region of multiple roots disappears, and above a certain threshold value of $\varepsilon$ there is a unique root of $\nabla R_{m 0}=0$ for any value of $\gamma$. From this figure it is seen that the threshold value is somewhere in the interval $0.01<\varepsilon<0.03$.

We now give an independent verification of the result of Proposition 3.2 for the roots of $\nabla R_{m 0}=0$. This analysis provides an alternative approach to the straightforward, but lengthy, calculation given above, which was based on the residue formula (3.7).

Consider two non-overlapping domains joined together by a very narrow channel on a sub-scale of size $\varepsilon$. Therefore, the outer problem consists of two domains $\Omega_{L}$ and $\Omega_{R}$ joined together at some point $y=\sigma$. We denote $V_{L}=\left|\Omega_{L}\right|$ and $V_{R}=\left|\Omega_{R}\right|$ as the areas of $\Omega_{L}$ and $\Omega_{R}$, and we set $V=V_{L}+V_{R}$. Let $G_{m}\left(y ; y_{0}\right)$ be the Neumann Green's function for $\Omega=\Omega_{L} \cup \Omega_{R} \cup\{\sigma\}$, satisfying

$$
\Delta G_{m}=\frac{1}{V}-\delta\left(y-y_{0}\right), \quad y \in \Omega ; \quad \partial_{n} G_{m}=0, \quad y \in \partial \Omega_{L} \cup \partial \Omega_{R},
$$

with $\int_{\Omega} G_{m} d y=0$. Now let $g_{m}\left(y ; y_{0}\right)$ be the Neumann Green's function for $\Omega_{R}$, so that

$$
\Delta g_{m}=\frac{1}{V_{R}}-\delta\left(y-y_{0}\right), \quad y \in \Omega_{R} ; \quad \partial_{n} g_{m}=0, \quad y \in \partial \Omega_{R} \backslash\{\sigma\},
$$

with $\int_{\Omega_{R}} g_{m} d y=0$. We then write $G_{m}=g_{m}+u$. For $y \in \Omega_{R}$, we obtain, up to some constant, that

$$
\Delta u=\frac{1}{V}-\frac{1}{V_{R}}, \quad y \in \Omega_{R} ; \quad \partial_{n} u=0, \quad y \in \partial \Omega_{R} \backslash\{\sigma\} .
$$

Since there is a singularity at the joining point $\sigma$, we look for a solution to (3.22) in the 
form $u=\alpha g_{m}(y ; \sigma)$, where $\alpha$ is to be found. A simple calculation using (3.21) and (3.22) determines $\alpha$ as $\alpha=-V_{L} /\left(V_{L}+V_{R}\right)$. Therefore,

$$
G_{m}\left(y ; y_{0}\right)=g_{m}\left(y ; y_{0}\right)-\frac{V_{L}}{V_{L}+V_{R}} g_{m}(y ; \sigma) .
$$

We define the regular parts $R_{m}\left(y ; y_{0}\right)$ and $r_{m}\left(y ; y_{0}\right)$ of $G_{m}$ and $g_{m}$, respectively, as

$$
G_{m}\left(y ; y_{0}\right)=-\frac{1}{2 \pi} \log \left|y-y_{0}\right|+R_{m}\left(y ; y_{0}\right), \quad g_{m}\left(y ; y_{0}\right)=-\frac{1}{2 \pi} \log \left|y-y_{0}\right|+r_{m}\left(y ; y_{0}\right) .
$$

Therefore, from (3.23) we get

$$
R_{m}\left(y ; y_{0}\right)=r_{m}\left(y ; y_{0}\right)-\frac{V_{L}}{V_{L}+V_{R}} g_{m}(y ; \sigma) .
$$

Suppose now that $\Omega_{R}$ is the unit ball centred at the origin and that $y_{0} \in \Omega_{R}$. We also assume that $\Omega_{L}$ is joined to $\Omega_{R}$ at the point $\sigma=(-1,0)$. For the unit disk $|y| \leqslant 1$ the functions $r_{m}\left(y ; y_{0}\right)$ and $g_{m}(y ; \sigma)$ can be calculated explicitly. The results, given below in $(4.3 b)$, yield

$$
\begin{aligned}
& r_{m}\left(y ; y_{0}\right)=\frac{1}{2 \pi}\left[-\log || y_{0}\left|y-\frac{y_{0}}{\left|y_{0}\right|}\right|+\frac{1}{2}\left(|y|^{2}+\left|y_{0}\right|^{2}\right)-\frac{3}{4}\right], \\
& g_{m}(y ; \sigma)=\frac{1}{2 \pi}\left[\frac{|y|^{2}}{2}-2 \log |y+1|-\frac{1}{4}\right] .
\end{aligned}
$$

We substitute (3.26) into (3.25) and calculate $\left.\nabla R_{m}\left(y ; y_{0}\right)\right|_{y=y_{0}} \equiv \nabla R_{m 0}$ to obtain

$$
\nabla R_{m 0}=\frac{1}{2 \pi}\left[y_{0}\left(\frac{\left|y_{0}\right|^{2}-2}{\left|y_{0}\right|^{2}-1}\right)-\frac{V_{L}}{V_{L}+V_{R}}\left(y_{0}-\frac{2}{\bar{y}_{0}+1}\right)\right] .
$$

Setting $\nabla R_{m 0}=0$, and taking $y_{0}$ to be real, we obtain that

$$
y_{0}\left(\frac{2-y_{0}^{2}}{1-y_{0}^{2}}\right)=\frac{V_{L}}{V_{L}+V_{R}}\left(y_{0}-\frac{2}{y_{0}+1}\right) .
$$

Next, we write $V_{R} / V_{L}=\gamma^{2}$. This formula is precisely the ratio between the two areas of the lobes of the dumbbell under the mapping (3.6) when $\varepsilon \ll 1$. Then, from (3.28), we obtain that $\nabla R_{m 0}=0$ when

$$
\gamma^{2}=\frac{2-y_{0}}{y_{0}\left(y_{0}^{2}-2\right)}
$$

This expression agrees identically with the relationship derived earlier in (3.18) from the residue formula (3.7).

Finally, note that when $y_{0} \rightarrow \sigma^{+}$, we can expand $\nabla R_{m 0}$ to obtain

$$
\nabla R_{m 0} \sim \frac{1}{2 \pi} \frac{y_{0}-\sigma}{\left|y_{0}-\sigma\right|^{2}}\left(\frac{1}{2}-2 \frac{V_{L}}{V_{L}+V_{R}}\right)+O(1), \quad y_{0} \rightarrow \sigma^{+} .
$$

Therefore, $\nabla R_{m 0} \rightarrow \pm \infty$ depending on whether $\frac{V_{L}}{V_{L}+V_{R}}$ is above or below $\frac{1}{4}$. The threshold 
value $\frac{V_{L}}{V_{L}+V_{R}}=\frac{1}{4}$ corresponds precisely to the threshold $\gamma^{2}=3$ as found in Proposition 3.2, when the root $x_{0}$ of $\nabla R_{m 0}=0$ near the neck of the dumbbell disappears. In fact it is not hard to show that this result depends only on the areas $V_{L}, V_{R}$ of the two domains, and not on their shape.

\section{Multiple holes in the unit disk: one ring configurations}

Let $\Omega$ be the unit circle, so that $|\Omega|=\pi$. For this domain, the Neumann Green's function $G_{m}$ in (1.5) has the form (cf. $\left.[4,30]\right)$

$$
G_{m}(x ; \xi)=\frac{1}{2 \pi}\left(-\log |x-\xi|-\log |x| \xi\left|-\frac{\xi}{|\xi|}\right|+\frac{1}{2}\left(|x|^{2}+|\xi|^{2}\right)+C(\xi)\right),
$$

for some $C(\xi)$. To determine $C$, we multiply the equation for $G_{m}$ in $(1.5 a)$ by $G_{m}\left(x ; \xi^{\prime}\right)$ and integrate over the unit disk $\Omega$. Then, by using $\int_{\Omega} G_{m}\left(x ; \xi^{\prime}\right) d x=0$ and integrating by parts with $\partial_{n} G_{m}=0$ on $\partial \Omega$, we get

$$
G_{m}\left(\xi ; \xi^{\prime}\right)=-\int_{\Omega} G_{m}\left(x ; \xi^{\prime}\right) \Delta G_{m}(x ; \xi) d x=\int_{\Omega} \nabla G_{m}\left(x ; \xi^{\prime}\right) \cdot \nabla G_{m}(x ; \xi) d x .
$$

This shows that $G_{m}\left(\xi ; \xi^{\prime}\right)=G_{m}\left(\xi^{\prime} ; \xi\right)$. From this relation and $(4.1)$ we obtain $C(\xi)=C\left(\xi^{\prime}\right)$, so that $C(\xi)=C$ is constant. To determine $C$, we evaluate (4.1) with source point at $\xi=0$, and we integrate $G_{m}(x ; 0)$ over $\Omega$ using $\int_{\Omega} G_{m}(x ; 0) d x=0$. Then, since $\int_{\Omega} \log |x| d x=-\pi / 2$ and $\int_{\Omega}|x|^{2} d x=\pi / 2$, we get $C=-3 / 4$. Therefore, for the unit circle, the Neumann Green's function $G_{m}$ and its regular part $R_{m}$ from $(1.5 b)$ are given explicitly by

$$
\begin{aligned}
& G_{m}(x ; \xi)=\frac{1}{2 \pi}\left(-\log |x-\xi|-\log |x| \xi\left|-\frac{\xi}{|\xi|}\right|+\frac{1}{2}\left(|x|^{2}+|\xi|^{2}\right)-\frac{3}{4}\right) \\
& R_{m}(\xi ; \xi)=\frac{1}{2 \pi}\left(-\log |\xi| \xi\left|-\frac{\xi}{|\xi|}\right|+|\xi|^{2}-\frac{3}{4}\right) .
\end{aligned}
$$

We now optimize $p$ in (2.25) for certain patterns of identical holes in the unit disk. To calculate the entries in the matrix $\mathscr{G}$ in $(2.20 c)$, it is convenient to represent points in the unit disk as complex numbers. The following simple formula is central to our analysis:

Lemma 4.1: Let $N>0$ and $n$ be integers, and $i \equiv \sqrt{-1}$. Then, for $y>0$, we have

$$
\prod_{j=1}^{N}\left(x-y e^{2 \pi i(j-n) / N}\right)=x^{N}-y^{N}
$$

Proof: Consider the polynomial $q(z)=z^{N}-w$, where $w=|w| e^{i \theta}$. The $N$ roots for $q(z)$ are $z=|w|^{1 / N} e^{i(\theta+2 \pi j) / N}$, for $j=1, \ldots, N$. This yields the factored polynomial

$$
z^{N}-|w| e^{i \theta}=\prod_{j=1}^{N}\left(z-|w|^{1 / N} e^{i(\theta+2 \pi j) / N}\right)
$$

Now suppose that $w>0$ and real, so that we can write $|w|=y^{N}$ for $y>0$ and $\theta=-2 \pi n$, where $n$ is an integer. Then, (4.5) reduces to (4.4). 
Consider a pattern with $N$ identical holes located symmetrically inside the unit disk as

$$
x_{j}=r e^{2 \pi i j / N}, \quad j=1, \ldots, N, \quad(\text { Pattern } \mathrm{I}),
$$

for some ring radius $r>0$, with $0<r<1$, and $N>1$. We will optimize $p$ in (2.25) with respect to $r$.

With Lemma 4.1 we readily obtain the following result:

Lemma 4.2: Let $N>1$ be an integer and let $x_{j}$ for $j=1, \ldots, N$ satisfy (4.6). Then, we have

$$
\begin{gathered}
\sum_{j=1}^{N} \log \left|x_{j}\right| x_{k}\left|-\frac{x_{k}}{\left|x_{k}\right|}\right|=\log \left(1-r^{2 N}\right) ; \quad \sum_{j=1}^{N}\left(\left|x_{j}\right|^{2}+\left|x_{k}\right|^{2}\right)=2 N r^{2} \\
\sum_{\substack{j=1 \\
j \neq k}}^{N} \log \left|x_{j}-x_{k}\right|=\log \left(N r^{N-1}\right) .
\end{gathered}
$$

Proof: To prove the first result in (4.7), we let $x_{j}=r e^{2 \pi i j / N}$ and then use Lemma 4.1 to calculate

$$
\begin{aligned}
& \sum_{j=1}^{N} \log \left|x_{j}\right| x_{k}\left|-\frac{x_{k}}{\left|x_{k}\right|}\right| \\
& \quad=\sum_{j=1}^{N} \log \left|r^{2} e^{2 \pi i j / N}-e^{2 \pi i k / N}\right|=\log \left|\prod_{j=1}^{N}\left(1-r^{2} e^{2 \pi i(j-k) / N}\right)\right|=\log \left(1-r^{2 N}\right) .
\end{aligned}
$$

The proof of the second result in (4.7) is immediate since $\left|x_{j}\right|^{2}=r^{2}$. Finally, we prove the third result in (4.7). We first write that

$$
\sum_{\substack{j=1 \\ j \neq k}}^{N} \log \left|x_{j}-x_{k}\right|=\log \left|\prod_{\substack{j=1 \\ j \neq k}}^{N}\left(r e^{2 \pi i j / N}-r e^{2 \pi i k / N}\right)\right|=\log \left|\prod_{\substack{j=1 \\ j \neq k}}^{N}\left(r-r e^{2 \pi i(j-k) / N}\right)\right|
$$

Then, using Lemma 4.1 we obtain

$$
\log \left|\prod_{\substack{j=1 \\ j \neq k}}^{N}\left(x-y e^{2 \pi i(j-k) / N}\right)\right|=\log \left|\frac{x^{N}-y^{N}}{x-y}\right|=\log \left|x^{N-1}\left[1+\left(\frac{y}{x}\right)+\cdots+\left(\frac{y}{x}\right)^{N-1}\right]\right|
$$

By using (4.10) with $x=y=r$, and substituting the result into (4.9), we get the third result in (4.7).

Next, we use Lemma 4.2 to calculate $p$ in (2.25) explicitly. The result is as follows: 
Proposition 4.3:(One Ring) Let $N>1$ be an integer, and let $x_{j}$ satisfy (4.6). Then, $p=p(r)$ in (2.25) is given by

$$
p=\frac{1}{2 \pi}\left[-N \log \left(N r^{N-1}\right)-N \log \left(1-r^{2 N}\right)+r^{2} N^{2}-\frac{3 N^{2}}{4}\right]
$$

Proof: We substitute (4.3) into (2.25) to obtain

$$
p=\frac{1}{2 \pi} \sum_{k=1}^{N}\left[-\sum_{j=1}^{N} \log \left|x_{j}\right| x_{k}\left|-\frac{x_{k}}{\left|x_{k}\right|}\right|+\frac{1}{2} \sum_{j=1}^{N}\left(\left|x_{j}\right|^{2}+\left|x_{k}\right|^{2}\right)-\frac{3 N}{4}-\sum_{\substack{j=1 \\ j \neq k}}^{N} \log \left|x_{j}-x_{k}\right|\right]
$$

By using the formulae of Lemma 4.2 directly in (4.12) we get (4.11) for $p$.

Next, we optimize $p$ with respect to the ring radius $r$. Setting $p^{\prime}(r)=0$, and recalling (2.24) with $|\Omega|=\pi$, we obtain the following main result for a one-ring pattern:

Proposition 4.4:(One Ring) Let $\Omega$ be the unit disk, $N>1$ be an integer, and suppose that the centres $x_{j}$, for $j=1, \ldots, N$, of $N$ identical holes are located symmetrically on a ring of radius $r$ according to (4.6). Then, the first eigenvalue $\lambda_{0}(\varepsilon)$ of (1.1) is given asymptotically by

$$
\lambda_{0}(\varepsilon) \sim \lambda^{*}, \quad \lambda^{*}=2 N v-4 \pi v^{2} p(r)+O\left(v^{3}\right) .
$$

Here $v=-1 / \log \varepsilon$, and $p(r)$ is given in (4.11). The function $p(r)$ has a unique minimum at $r=r_{c}$, where $r_{c}$ is the root of the transcendental equation

$$
\frac{r^{2 N}}{1-r^{2 N}}=\frac{N-1}{2 N}-r^{2}
$$

For Pattern I, the ring radius $r_{c}$ maximizes $\lambda_{0}(\varepsilon)$ through terms of order $v^{2}$.

The fact that there is a unique $r=r_{c}$ where $p^{\prime}(r)=0$ is seen from (4.14). Since the left-hand side of (4.14) is monotonically increasing in $0<r<1$, while the right-hand side of (4.14) is monotonically decreasing, it follows that there is a unique $r=r_{c}$ in $0<r_{c}<\sqrt{(N-1) /(2 N)}$ where $p^{\prime}(r)=0$. A simple calculation then shows that $p^{\prime \prime}\left(r_{c}\right)>0$, so that $r_{c}$ does indeed minimize $p(r)$.

Next, we construct a different pattern of $N$ identical holes. We consider $N-1$ holes symmetrically located on a ring of radius $r$, with one hole located at the origin. The centres of the holes satisfy

$$
x_{j}=r e^{2 \pi i j /(N-1)}, \quad j=1, \ldots, N-1 ; \quad x_{N}=0, \quad \text { (Pattern II) } .
$$

For this pattern, the following result is obtained in place of Lemma 4.2: 
Lemma 4.5: Let $N>1$ be an integer, and let $x_{j}$ for $j=1, \ldots, N$ satisfy (4.15). Then, we have

$$
\begin{gathered}
\sum_{j=1}^{N} \log \left|x_{j}\right| x_{k}\left|-\frac{x_{k}}{\left|x_{k}\right|}\right|=\log \left(1-r^{2(N-1)}\right)\left(1-\delta_{k, N}\right), \\
\sum_{k=1}^{N} \sum_{j=1}^{N}\left(\left|x_{j}\right|^{2}+\left|x_{k}\right|^{2}\right)=2 N(N-1) r^{2} ; \\
\sum_{\substack{j=1 \\
j \neq k}}^{N} \log \left|x_{j}-x_{k}\right|= \begin{cases}(N-1) \log r, & k=N, \\
\log r+\log \left((N-1) r^{N-2}\right), & k \neq N .\end{cases}
\end{gathered}
$$

Here $\delta_{j, k}$ is the Kronecker symbol, with $\delta_{k, k}=1$ and $\delta_{j, k}=0$ if $j \neq k$.

Proof: The proof is similar to that of Lemma 4.2 and is omitted. Here attention is needed since $x_{N}=0$.

By using (4.3), together with the formulae of Lemma 4.5, we obtain the following result for $p$ :

Proposition 4.6:(One Ring and a Centre Hole) Let $N>1$ be an integer, and let $x_{j}$ satisfy (4.15). Then, $p=p(r)$ in (2.25) is given by

$$
p=\frac{1}{2 \pi}\left[-(N-1) \log \left[(N-1) r^{N}\right]-(N-1) \log \left(1-r^{2(N-1)}\right)+r^{2} N(N-1)-\frac{3 N^{2}}{4}\right] \text {. }
$$

Finally, we optimize $p$ with respect to the ring radius $r$. Setting $p^{\prime}(r)=0$, we obtain an equation for $r=r_{c}$ that has a unique root $r_{c}$ in $0<r<1$, with $p^{\prime \prime}\left(r_{c}\right)>0$. This leads to the following main result for Pattern II:

Proposition 4.7:(One Ring and a Centre Hole) Let $\Omega$ be the unit disk, $N>1$ be an integer, and suppose that the centres $x_{j}$, for $j=1, \ldots, N$ of $N$ identical holes satisfy (4.15). Then, the first eigenvalue $\lambda_{0}(\varepsilon)$ of (1.1) is given asymptotically by (4.13), where $p=p(r)$ is given by (4.17). This function has a unique minimum in $0<r<1$ at $r=r_{c}$, where $r_{c}$ satisfies

$$
\frac{r^{2 N-2}}{1-r^{2 N-2}}=\frac{N}{N-1}\left(\frac{1}{2}-r^{2}\right)
$$

For Pattern II, the ring radius $r_{c}$, satisfying $r_{c}^{2}<1 / 2$, maximizes $\lambda_{0}(\varepsilon)$ through terms of order $v^{2}$.

In Table 1 we give numerical results for the optimum ring radius $r_{c}$ and the minimum value $p_{c}=p\left(r_{c}\right)$ for both Patterns I and II. In Fig. 4(a) we plot $p$ versus $r$ for Pattern I, corresponding to 2,3 , and 4 , holes on a ring. For these values of $N$, a plot of $\lambda_{0}(\varepsilon)$ versus $\varepsilon$ at the optimum ring radius is shown in Fig. 4. A similar plot is shown in Fig. 5 for Pattern II. From Table 1 we observe that Pattern II gives a smaller value for $p$ than Pattern I, and hence a larger $\lambda_{0}(\varepsilon)$, only when $N \geqslant 7$. In Fig. 6 we plot $p$ versus $r$ for Pattern 
Table 1. Numerical results for the optimum radius $r_{c}$ and the minimum value $p_{c} \equiv p\left(r_{c}\right)$. The second and third columns are for Pattern I where $N$ holes are on a ring. The fourth and fifth columns are for Pattern II, where $N-1$ holes are on a ring, and one hole is at the origin

\begin{tabular}{rcccc}
\hline \hline$N$ & $r_{c}$ & $p_{c}$ & $r_{c}$ & $p_{c}$ \\
\hline 2 & 0.4536 & -0.3017 & 0.5412 & -0.1336 \\
3 & 0.5516 & -0.5813 & 0.6200 & -0.4205 \\
4 & 0.5986 & -0.8893 & 0.6584 & -0.7677 \\
5 & 0.6252 & -1.2073 & 0.6796 & -1.1374 \\
6 & 0.6416 & -1.5260 & 0.6918 & -1.5134 \\
7 & 0.6528 & -1.8398 & 0.6988 & -1.8871 \\
8 & 0.6604 & -2.1451 & 0.7026 & -2.2538 \\
9 & 0.6662 & -2.4391 & 0.7048 & -2.6104 \\
10 & 0.6706 & -2.7200 & 0.7058 & -2.9549 \\
\hline \hline
\end{tabular}

(a)

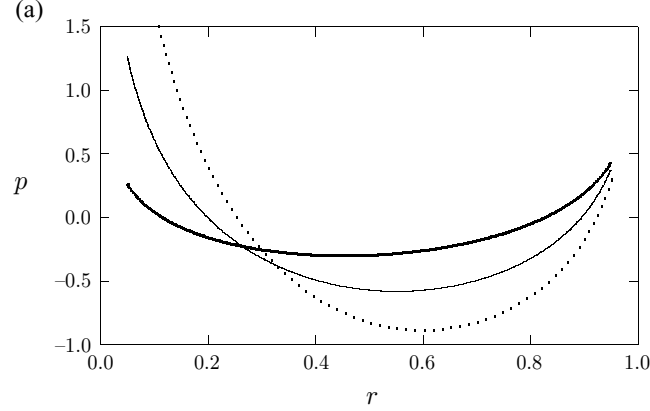

(b)

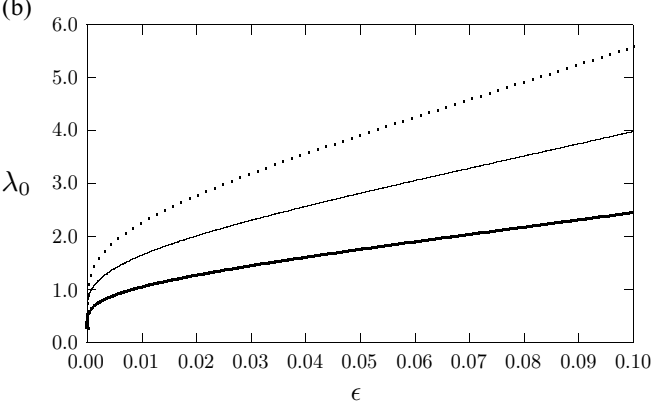

Figure 4. (a): Plots of $p(r)$, given in (4.11), for $N=2,3,4$ holes on a ring. (b): Plots of $\lambda_{0}$ versus $\varepsilon$ in (4.13) at the optimum ring radius. Here $N=2$ (heavy solid curve), $N=3$ (solid curve), and $N=4$ (dotted curve).

(a)

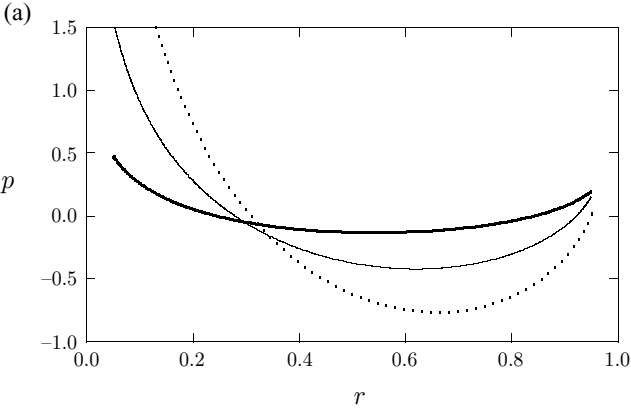

(b)

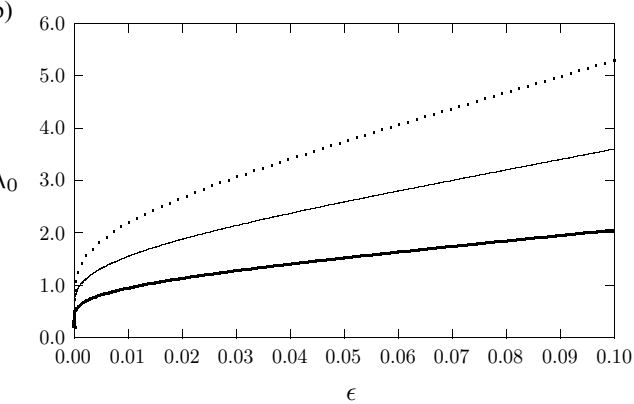

FIGURE 5. (a): Plots of $p(r)$, given in (4.17), for holes on a ring together with a centre hole. (b): Plots of $\lambda_{0}$ versus $\varepsilon$ in (4.13) at the optimum ring radius. The labels are $N=2$ (heavy solid curve), $N=3$ (solid curve), and $N=4$ (dotted curve). 


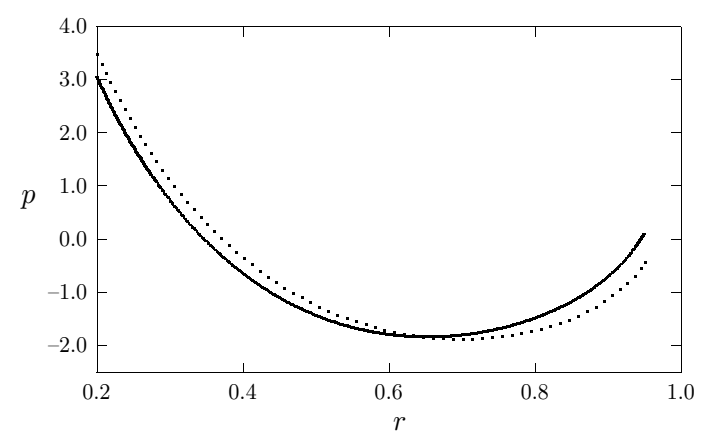

Figure 6. Plot of $p$ versus $r$ for 7 holes on a ring (heavy solid curve), and 6 holes on a ring together with a centre hole (dotted curve). Notice that the configuration with a centre hole gives a slightly smaller optimum value for $p$.

I consisting of 7 ring holes and for Pattern II consisting of 6 ring holes and a centre hole. The second pattern provides a marginally smaller value of $p$ at the optimum ring radius.

Next, we consider the limit $N \gg 1$, with $N \varepsilon \ll 1$. For both patterns it is easy to see from (4.14) and (4.18) that $r_{c} \rightarrow 1 / \sqrt{2}$ for $N \gg 1$. Hence, for $N \gg 1$, the optimum value $r_{c}=1 / \sqrt{2}$ is the one for which the holes on the ring separate the unit disk into two regions each of area $A=\pi / 2$.

The results of Propositions 4.3 and 4.6 for Patterns I and II have a natural interpretation in terms of spectral properties of the matrix $\mathscr{G}$. Recall that $p$ was given in terms of the eigenvalues and eigenvectors of $\mathscr{G}$ in (2.26). We begin by stating a lemma concerning the spectrum of an arbitrary $N \times N$ symmetric and cyclic matrix.

Lemma 4.8:(Cyclic and Symmetric Matrix) Consider the $N \times N$ cyclic matrix $\mathscr{M}$ defined by

$$
\mathscr{M} \equiv\left(\begin{array}{ccccc}
a_{1} & a_{2} & a_{3} & \cdots & a_{N} \\
a_{N} & a_{1} & a_{2} & \cdots & a_{N-1} \\
a_{N-1} & a_{N} & a_{1} & \cdots & a_{N-2} \\
\vdots & & & \ddots & \vdots \\
a_{2} & a_{3} & a_{4} & \cdots & a_{1}
\end{array}\right) .
$$

Assume also that $\mathscr{M}$ is a symmetric matrix, so that $a_{2+j}=a_{N-j}$ for $j=0, \ldots, N-2$. Then, the eigenvalues $\chi_{j}$ and the (unnormalized) eigenvectors $\boldsymbol{v}_{j}$ of $\mathscr{M}$, for $j=1, \ldots, N$, are given by

$$
\begin{gathered}
\chi_{j}=\sum_{k=0}^{N-1} a_{k+1} \cos \left(\frac{2 \pi(j-1) k}{N}\right) \\
\boldsymbol{v}_{j}^{t}=\left(1, \cos \left(\frac{2 \pi(j-1)}{N}\right), \ldots, \cos \left(\frac{2 \pi(j-1)(N-1)}{N}\right)\right) .
\end{gathered}
$$


Proof: Let $z^{N}=1$, so that $z=e^{2 \pi i j / N}$, for $j=0, \ldots, N-1$. Define $\boldsymbol{v}$ by $\boldsymbol{v}^{t}=$ $\left(1, z, z^{2}, \ldots, z^{N-1}\right)$. Then, a direct calculation shows that $\mathscr{M} \boldsymbol{v}=\chi \boldsymbol{v}$, where

$$
\chi=\sum_{k=0}^{N-1} a_{k+1} z^{k}
$$

Then, using the symmetry of the matrix $\mathscr{M}$, so that $a_{N-j}=a_{2+j}$ for $j=0, \ldots, N-2$, and the $N$ possible values for $z$, we obtain that (4.21) reduces to the expression for $\chi_{j}$ in (4.20). Since $\mathscr{M}$ is symmetric, the eigenvectors are found by taking the real part of $\boldsymbol{v}^{t}=\left(1, z, z^{2}, \ldots, z^{N-1}\right)$.

For Pattern I, given by (4.6), it is easy to see that $\mathscr{G}$ is a symmetric and cyclic matrix. Therefore, from (4.20), we conclude that $s_{1}^{t}=N^{-1 / 2}(1, \ldots, 1)$ is an eigenvector of $\mathscr{G}$ for any ring radius $r$. By the orthogonality of the eigenvectors of $\mathscr{G}$, it follows that the sum in (2.26) collapses to the one term $p\left(x_{1}, \ldots, x_{N}\right)=N \mu_{1}$. Here $\mu_{1}$ is the eigenvalue of $\mathscr{G}$ associated with $s_{1}$. From (4.20), $\mu_{1}$ corresponds to $\chi_{1}$, and hence

$$
\mu_{1}=R_{m}\left(x_{1} ; x_{1}\right)+\sum_{j=2}^{N} G_{m}\left(x_{1} ; x_{j}\right) .
$$

Therefore, the optimization procedure for $N$ holes symmetrically placed on a ring is equivalent to determining the critical ring radius $r_{c}$ that minimizes $\mu_{1}$.

To obtain a similar spectral characterization for Pattern II we need the following modification of Lemma 4.8 .

Lemma 4.9:(Symmetric and Near Cyclic Matrix) Consider the $N \times N$ near-cyclic matrix $M$ defined by

$$
\mathscr{M} \equiv\left(\begin{array}{cccccc}
a_{1} & a_{2} & a_{3} & \cdots & a_{N-1} & \alpha \\
a_{N-1} & a_{1} & a_{2} & \cdots & a_{N-2} & \alpha \\
a_{N-2} & a_{N-1} & a_{1} & \cdots & a_{N-3} & \alpha \\
\vdots & & & \ddots & \vdots & \alpha \\
a_{2} & a_{3} & a_{4} & \cdots & a_{1} & \alpha \\
\alpha & \alpha & \alpha & \cdots & \alpha & \beta
\end{array}\right) .
$$

Assume also that $\mathscr{M}$ is a symmetric matrix, so that $a_{2+j}=a_{N-j-1}$ for $j=0, \ldots, N-3$. Then, the eigenvalues $\chi_{j}$ and the unnormalized eigenvectors $\boldsymbol{v}_{j}$ of $\mathscr{M}$ are given by

$$
\chi_{j}=\sum_{k=0}^{N-2} a_{k+1} \cos \left(\frac{2 \pi j k}{N-1}\right), \quad \boldsymbol{v}_{j}=\left(\begin{array}{c}
\cos (2 \pi j /(N-1)) \\
\vdots \\
\cos (2 \pi j(N-2) /(N-1)) \\
0
\end{array}\right), \quad j=1, \ldots, N-2 .
$$


The other two eigenpairs $\boldsymbol{v}_{ \pm}$and $\chi_{ \pm}$are given by

$$
\chi_{ \pm}=\alpha \gamma_{ \pm}+\zeta, \quad \boldsymbol{v}_{ \pm}^{t}=\left(1, \ldots, 1, \gamma_{ \pm}\right)
$$

where $\gamma_{ \pm}$and $\zeta$ are defined by

$$
\gamma_{ \pm} \equiv-\frac{(\zeta-\beta)}{2 \alpha} \pm\left[\frac{(\zeta-\beta)^{2}}{4 \alpha^{2}}+(N-1)\right]^{1 / 2}, \quad \zeta \equiv \sum_{k=1}^{N-1} a_{k}
$$

Proof: Let $z^{N-1}=1$, and define $\boldsymbol{v}^{t}=\left(1, z, \ldots, z^{N-2}, 0\right)$. Since $\sum_{j=0}^{N-2} \alpha z^{j}=\alpha\left(z^{N-1}-1\right) /(z-$ $1)=0$, we can proceed as in the proof of Lemma 4.8 to obtain (4.24). To prove (4.25) we let $\boldsymbol{v}^{t}=(1, \ldots, 1, \gamma)$ and we calculate that $\mathscr{M} \boldsymbol{v}=\lambda \boldsymbol{v}$, provided that $\lambda$ and $\gamma$ satisfy the coupled system

$$
(N-1) \alpha+\beta \gamma=\lambda \gamma, \quad \zeta+\alpha \gamma=\lambda .
$$

Solving (4.26) for $\lambda$ and $\gamma$ we obtain (4.25).

For Pattern II, where the hole locations satisfy (4.15), it is easy to see that $\mathscr{G}$ has the form of the matrix in Lemma 4.9, where $\alpha$ and $\beta$ are given by

$$
\alpha \equiv G\left(x_{j} ; 0\right)=\frac{1}{2 \pi}\left[-\log r+\frac{r^{2}}{2}-\frac{3}{4}\right], \quad \beta \equiv R_{m}(0 ; 0)=-\frac{3}{8 \pi} .
$$

The eigenvectors $\boldsymbol{v}_{j}$ for $j=1, \ldots, N-2$ in (4.24) are orthogonal to the vector $\boldsymbol{e}^{t}=$ $N^{-1 / 2}(1, \ldots, 1)$. Therefore, the sum in (2.26) collapses the sum of two terms

$$
p\left(x_{1}, \ldots, x_{N}\right)=\chi_{+} \frac{\left[(N-1)+\gamma_{+}\right]^{2}}{(N-1)+\gamma_{+}^{2}}+\chi_{-} \frac{\left[(N-1)+\gamma_{-}\right]^{2}}{(N-1)+\gamma_{-}^{2}} .
$$

Here $\chi_{ \pm}$and $\gamma_{ \pm}$are given in (4.25a) and (4.25b), where $\zeta \equiv R_{m}\left(x_{1} ; x_{1}\right)+\sum_{j=2}^{N-1} G_{m}\left(x_{1} ; x_{j}\right)$. Therefore, the optimization procedure for a ring with a centre hole is spectrally equivalent to minimizing (4.28).

In $\S 9$ of [28] (see Fig. 11 of [28]) numerical results were given for the solution $\lambda^{*}$ to (2.18) for two separate four-hole patterns in the unit disk. The hole locations for these patterns are either $x_{j}=\frac{1}{4} e^{\pi i(2 j+1) / 4}$ or $x_{j}=\frac{1}{2} e^{\pi i(2 j+1) / 4}$, for $j=0, \ldots, 3$. From (4.11), the values of $p$ in (4.13) are $p=.1439$ or $p=-.8295$, respectively. Recall that the solution, $\lambda^{*}$, to (2.18) provides an approximation to $\lambda_{0}$ that is asymptotically accurate to all logarithmic terms. In Fig. 7 we show a reasonably favorable comparison between the two-term result of (4.13) and the numerical results for $\lambda^{*}$, computed in [28], for each of these four-hole patterns.

\section{Multiple holes in the unit disk: More general configurations}

A similar analysis can be done for the case of two rings, where $K$ holes are on an inner ring of radius $r$, and $J$ holes are on an outer ring of radius $\rho>r$, so that

$$
\xi_{k}=r e^{2 \pi i k / K}, \quad k=1, \ldots, K ; \quad y_{j}=\rho e^{2 \pi i j / J} e^{i \phi}, \quad j=1, \ldots, J
$$




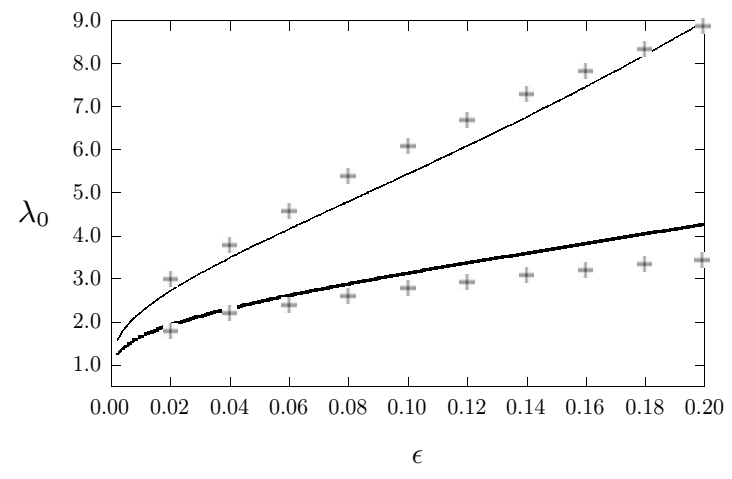

FIGURE 7. Plots of $\lambda_{0}$ versus $\varepsilon$, computed from the two-term asymptotic result (4.13), for a four-hole pattern with holes at $x_{j}=\frac{1}{4} e^{\pi i(2 j+1) / 4}$ (heavy solid curve) or at $x_{j}=\frac{1}{2} e^{\pi i(2 j+1) / 4}$ (solid curve), for $j=0, \ldots, 3$. The discrete points are the numerical results $\lambda^{*}$ of [28], computed from (2.18), that involve summing all the logarithmic terms in $\lambda_{0}$.

Here $\phi$ represents a uniform phase angle between the hole locations on the two rings. Collectively, the hole locations are arranged according to

$$
x_{n}= \begin{cases}\xi_{n}, & n=1, \ldots, K, \\ y_{n-K}, & n=K+1, \ldots, N .\end{cases}
$$

Here $N=J+K$ is the total number of holes. For this two-ring configuration, the following lemma allows for an explicit calculation of the matrix $\mathscr{G}$ in (2.25).

Lemma 5.1: Let $K>0$ and $J>0$ be positive integers, and $i \equiv \sqrt{-1}$. Then, for $y>0$, we have

$$
\prod_{k=1}^{K}\left(x-y e^{2 \pi i k J / K}\right)=\left(x^{K / g}-y^{K / g}\right)^{g},
$$

where $g=\operatorname{gcd}(J, K)$ is the greatest common divisor of $J$ and $K$.

Proof: Consider the polynomial $q(z)=z^{n}-w^{m}$, with $w=|w| e^{i \theta}$, where $m$ and $n$ are relatively prime positive integers. Then, we can factor the polynomial as

$$
z^{n}-w^{m}=\prod_{k=1}^{n}\left(z-|w|^{m / n} e^{i(\theta+2 \pi k) m / n}\right) .
$$

Now suppose that $J$ and $K$ are two positive integers. We write these integers as $J=g m$ and $K=g n$, where $m$ and $n$ are relatively prime integers and $g=\operatorname{gcd}(J, K)$. From (5.3), we then obtain

$$
\left(z^{K / g}-w^{J / g}\right)^{g}=\prod_{k=1}^{K}\left(z-|w|^{J / K} e^{i(\theta+2 \pi k) J / K}\right) .
$$

Finally, by substituting $z=x, w=y^{K / J}>0$ and $\theta=0$ into (5.4), we obtain (5.2).

With Lemma 5.1 we can derive the following result: 
Lemma 5.2: Let $N>1$ be an integer and let $x_{j}$ for $j=1, \ldots, N$ satisfy (4.6). Then, we have

$$
\begin{gathered}
\sum_{m=1}^{N} \sum_{n=1}^{N} \log \left|x_{m}\right| x_{n}\left|-\frac{x_{n}}{\left|x_{n}\right|}\right|=\log \left(1-r^{2 K}\right)+\log \left(1-\rho^{2 J}\right)+2 g \log \left|(r \rho)^{J K / g}-e^{i J K \phi / g}\right| \\
\frac{1}{2} \sum_{m=1}^{N} \sum_{n=1}^{N}\left(\left|x_{m}\right|^{2}+\left|x_{n}\right|^{2}\right)=K^{2} r^{2}+K J\left(r^{2}+\rho^{2}\right)+J^{2} \rho^{2} \\
\sum_{m=1}^{N} \sum_{\substack{n=1 \\
n \neq m}}^{N} \log \left|x_{m}-x_{n}\right|=K \log \left(K r^{K-1}\right)+J \log \left(J \rho^{J-1}\right)+2 g \log \left|r^{J K / g}-\rho^{J K / g} e^{i J K \phi / g}\right|
\end{gathered}
$$

Proof: For each of the terms above we decompose the double sum using the block structure of $\mathscr{G}$ as

$$
\sum_{m=1}^{N} \sum_{n=1}^{N} c_{m n}=\sum_{k=1}^{K} \sum_{k^{\prime}=1}^{K} c_{k k^{\prime}}+\sum_{j=1}^{J} \sum_{j^{\prime}=1}^{J} c_{j j^{\prime}}+2 \sum_{k=1}^{K} \sum_{j=1}^{J} c_{j k}
$$

where $c_{m n}$ is any one of the terms on the left-hand side of the expressions in (5.5). The first two terms on the right-hand side of (5.6) were calculated in Lemma 4.2. Therefore, we need only calculate the ring-interaction term in (5.6), represented by the last term on the right-hand side of (5.6).

We first establish (5.5a). By using (5.1 a), together with equation (4.5) of Lemma 4.1, we calculate

$$
\begin{aligned}
\sum_{k=1}^{K} \sum_{j=1}^{J} \log \left|\xi_{k}\right| y_{j}\left|-\frac{y_{j}}{\left|y_{j}\right|}\right| & =\sum_{k=1}^{K} \sum_{j=1}^{J} \log \left|(r \rho) e^{2 \pi i k / K}-e^{i \phi+2 \pi i j / J}\right| \\
& =\sum_{j=1}^{J} \log \left|\prod_{k=1}^{K}\left(1-(r \rho) e^{i(\theta+2 \pi k) / K}\right)\right| \\
& =\sum_{j=1}^{J} \log \left|1-(r \rho)^{K} e^{i \theta}\right|,
\end{aligned}
$$

where $\theta \equiv-K \phi-2 \pi j K / J$. We then use (5.3) of Lemma 5.1 to calculate the last expression in $(5.7)$ as

$$
\sum_{k=1}^{K} \sum_{j=1}^{J} \log \left|\xi_{k}\right| y_{j}\left|-\frac{y_{j}}{\left|y_{j}\right|}\right|=\log \left|\prod_{j=1}^{J}\left((r \rho)^{K}-e^{i(J \phi+2 \pi j) K / J}\right)\right|=g \log \left|(r \rho)^{J K / g}-e^{i \phi J K / g}\right| .
$$

Finally, by substituting (5.8) into the generic form (5.6), and by using the first result in (4.7) of Lemma 4.2 to evaluate the self-interaction term for each ring, we obtain $(5.5 a)$. 
Next, we prove (5.5c). By using (5.1 a) and (4.5) of Lemma 4.1, we calculate

$$
\begin{aligned}
\sum_{k=1}^{K} \sum_{j=1}^{J} \log \left|\xi_{k}-y_{j}\right| & =\sum_{j=1}^{J} \log \left|\prod_{k=1}^{K}\left(r e^{2 \pi i k / K}-\rho e^{i \phi+2 \pi i j / J}\right)\right|=\sum_{j=1}^{J} \log \left|\prod_{k=1}^{K}\left(\rho-r e^{i(\theta+2 \pi k) / K}\right)\right| \\
& =\sum_{j=1}^{J} \log \left|\rho^{K}-r^{K} e^{-i K \phi-2 \pi i j K / J}\right|
\end{aligned}
$$

where $\theta \equiv-K \phi-2 \pi j K / J$. We then use (5.3) of Lemma 5.1 to calculate the last expression in $(5.9)$ as

$$
\sum_{k=1}^{K} \sum_{j=1}^{J} \log \left|\xi_{k}-y_{j}\right|=\log \left|\prod_{j=1}^{J}\left(r^{K}-\rho^{K} e^{i(J \phi+2 \pi j) K / J}\right)\right|=g \log \left|r^{J K / g}-\rho^{J K / g} e^{i \phi J K / g}\right| .
$$

Then, by combining (5.10), the third result in (4.7), and (5.6), we obtain $(5.5 c)$. Finally, the proof of $(5.5 b)$ is straightforward since $\left|x_{m}\right|=r$ if $m=1, \ldots, K$, and $\left|x_{m}\right|=\rho$ if $m=K+1 \ldots, J+K$.

By using (4.3) and Lemma 5.2 to calculate $p$ in (2.25) explicitly, we obtain the following result:

Proposition 5.3:(Two Rings) Let $K$ and $J$ be positive integers, with $K$ holes on a ring of radius $r$ and $J$ holes on a ring of radius $\rho$, where the holes are aligned according to (5.1). Assume that $0<r<\rho<1$. Then, the function $p$ in (2.25) is given by $p=p_{*} /(2 \pi)$, where

$$
\begin{aligned}
p_{*} \equiv & -K \log \left(K r^{K-1}\right)-J \log \left(J \rho^{J-1}\right)-2 g \log \left|r^{J K / g}-\rho^{J K / g} e^{i J K \phi / g}\right| \\
& -K \log \left(1-r^{2 K}\right)-J \log \left(1-\rho^{2 J}\right)-2 g \log \left|(r \rho)^{J K / g}-e^{i J K \phi / g}\right| \\
& +K^{2} r^{2}+K J\left(r^{2}+\rho^{2}\right)+J^{2} \rho^{2}-\frac{3}{4}(J+K)^{2} .
\end{aligned}
$$

In a similar way, one can calculate $p$ for a two-ring configuration with a hole at the centre of the unit disk. For this configuration, (5.1) is replaced by

$$
x_{n}= \begin{cases}r e^{2 \pi i k / K} & n=1, \ldots, K \\ \rho e^{2 \pi i(n-K) / J+i \phi} & n=K+1, \ldots, J+K \\ 0 & n=N\end{cases}
$$

Here $N=J+K+1$ is the total number of holes. For this configuration, we have the following result:

Proposition 5.4:(Two Rings and a Centre Hole) Suppose that the two-ring configuration satisfies (5.12) with a centre hole at the origin. Then, with $p_{*}$ as defined in (5.11), the function $p$ in (2.25) is given by

$$
p=\frac{1}{2 \pi}\left[p_{*}-2(K \log r+J \log \rho)+K r^{2}+J \rho^{2}-\frac{3}{4}(2 K+2 J+1)\right] .
$$


We first optimize (5.11) with respect to the phase angle $\phi$. Since $\log \left|x-e^{-i w}\right| \leqslant \log |x+1|$ for any $x>0$ and $\omega$ real, it follows from (5.11) that $p_{*}$ is minimized for any $0<r<\rho$, when

$$
\phi=\frac{\pi g}{J K}, \quad g=\operatorname{gcd}(J, K)
$$

For example, $\phi=\pi / 3$ when $J=K=3$, and $\phi=\pi / 4$ when $K=2$, and $J=4$. With $\phi$ determined in this way, the stationary points of $p_{*}$, defined in (5.11), with respect to $r$ and $\rho$, are found to satisfy

$$
\begin{gathered}
-\frac{K(K-1)}{r}+\frac{2 K^{2} r^{2 K-1}}{1-r^{2 K}}+2 r\left(J K+K^{2}\right)-\frac{2 K J}{r}\left[\frac{(r / \rho)^{\beta}}{1+(r / \rho)^{\beta}}+\frac{(r \rho)^{\beta}}{1+(r \rho)^{\beta}}\right]=0 \\
-\frac{J(J-1)}{\rho}+\frac{2 J^{2} \rho^{2 J-1}}{1-\rho^{2 J}}+2 \rho\left(J K+K^{2}\right)-\frac{2 K J}{\rho}\left[\frac{1}{1+(r / \rho)^{\beta}}+\frac{(r \rho)^{\beta}}{1+(r \rho)^{\beta}}\right]=0
\end{gathered}
$$

where $\beta$ is defined by $\beta=K J / g$. For a pattern with two rings and a hole at the centre, we must add the terms $2 K r-2 K / r$ and $2 J \rho-2 J / \rho$ to the right-hand sides of $(5.15 a)$ and $(5.15 b)$, respectively.

We now consider a limiting configuration of two rings where $J=K \gg 1$. Since $0<$ $r<\rho<1$, (5.15) reduces in this limit to $4 r^{2}=1$ and $4 \rho=3 / \rho$, so that $r=1 / 2$, and $\rho=\sqrt{3} / 2$. The geometrical interpretation of this result is that the unit disk is partitioned into three regions $A_{1}: 0 \leqslant|x| \leqslant r, A_{2}: r \leqslant|x| \leqslant \rho$, and $A_{3}: \rho \leqslant|x| \leqslant 1$, with areas $A_{1}=\pi / 4, A_{2}=\pi / 2$, and $A_{3}=\pi / 4$. The result that $A_{2}$ is twice as large as the other areas is a reflection of the fact that this region is bounded by two rings of traps, whereas $A_{1}$ and $A_{3}$ each have only one ring of traps.

A different limiting result is obtained if the number of holes is allowed to scale with the circumference of the ring, so that $K=\alpha r$ and $J=\alpha \rho$, with $\alpha \gg 1$. Then, from (5.15), we obtain

$$
r-2 r\left(\rho r+r^{2}\right)=0, \quad-\rho+2 \rho\left(\rho r+\rho^{2}\right)=2 r
$$

which has the unique solution $r=1 / \sqrt{6}$ and $\rho=\sqrt{6} / 3$. This limiting configuration partitions the unit disk into three regions of areas $A_{1}=\pi / 6, A_{2}=\pi / 2$, and $A_{3}=\pi / 3$. It is straightforward to generalize the two-ring pattern to the case where we have $m>2$ rings. For this case, we obtain the following generalization of Proposition 5.3.

Proposition 5.5:( $m$ rings) Consider $m$ rings of radii $r_{1}, \ldots, r_{m}$, with $r_{j}<r_{j+1}$, inside the unit disk. Assume that there are $J_{k}$ holes on the ring of radius $r_{k}$. On the $k$ th ring, for $k=1, \ldots, m$, the centres of the holes are assumed to satisfy

$$
\xi_{j}^{(k)}=r_{k} e^{2 \pi i j / J_{k}} e^{i \phi_{k}}, \quad j=1, \ldots, J_{k}
$$

Here $\phi_{k}$ is a phase angle with $\phi_{1}=0$. For this configuration, the function $p$ in (2.25) is 
given by $p=p_{*} /(2 \pi)$, where $p_{*}$ is given by

$$
\begin{aligned}
p_{*} \equiv & -\sum_{k=1}^{m} J_{k} \log \left(J_{k} r^{J_{k}-1}\right)-\sum_{k=1}^{m} \sum_{k^{\prime}=1}^{m} g_{k, k^{\prime}} \log \left|\left(r_{k} r_{k^{\prime}}\right)^{\beta_{k, k^{\prime}}}-e^{i \beta_{k, k^{\prime}} \Delta_{k, k^{\prime}}}\right|+\sum_{k=1}^{m} J_{k}^{2} r_{k}^{2} \\
& -2 \sum_{k=1}^{m} \sum_{\substack{k^{\prime}=1 \\
k<k^{\prime}}}^{m} g_{k, k^{\prime}} \log \left|r_{k}^{\beta_{k, k^{\prime}}}-r_{k^{\prime}}^{\beta_{k, k^{\prime}}} e^{i \beta_{k, k^{\prime}} \Delta_{k, k^{\prime}}}\right|-\frac{3}{4}\left(\sum_{k=1}^{m} J_{k}\right)^{2}+\frac{1}{2} \sum_{k=1}^{m} \sum_{k^{\prime}=1}^{m} J_{k} J_{k^{\prime}}\left(r_{k}^{2}+r_{k^{\prime}}^{2}\right) .
\end{aligned}
$$

Here $\Delta_{k, k^{\prime}}, \beta_{k, k^{\prime}}$, and $g_{k, k^{\prime}}$ are defined by $\Delta_{k, k^{\prime}}=\phi_{k^{\prime}}-\phi_{k}, \beta_{k, k^{\prime}}=\frac{J_{k} J_{k^{\prime}}}{g_{k, k^{\prime}}}$, and $g_{k, k^{\prime}}=\operatorname{gcd}\left(J_{k}, J_{k^{\prime}}\right)$. If, in addition to having $m$ rings, we inserted a hole at the centre of the unit disk, then $p$ in (2.25) is given by

$$
p=\frac{1}{2 \pi}\left(p_{*}-2 \sum_{k=1}^{m} J_{k} \log r_{k}+\sum_{k=1}^{m} J_{k} r_{k}^{2}-\frac{3}{2} \sum_{k=1}^{m} J_{k}-\frac{3}{4}\right) .
$$

Within the class of two-ring and three-ring patterns of the form (5.1) and (5.17), with possibly an additional centre hole, we now give some numerical results for the minimum value of $p$ (and hence the maximum $\lambda_{0}$ ) for patterns up to a total of 25 holes. To display our results we introduce the notation $\left(j_{1}, \ldots, j_{m}\right)$, to indicate an optimum $m$-ring pattern with $j_{q}$ holes on the ring $r_{q}$, where $r_{q}<r_{q+1}$. If the minimum value of $p$ is obtained with an $m$-ring pattern and a centre hole, we denote the optimum pattern by $[1]\left(j_{1}, \ldots, j_{m}\right)$. The results, shown in Table 2, are obtained by using Newton's method on (5.15) and (5.18) to compute the optimum ring radii. For the optimum three-ring pattern shown in the last row of Table 2 we have chosen the phase angles $\phi_{k}=0$ in (5.18), for $k=1,2,3$. Other choices for the phase angles for the $(2,8,15)$ pattern do not change the results for $p$ up to at least four significant digits. In fact, many of the results shown in Table 2 are rather insensitive to the choice of the phase angle. This is clear from examining the terms in (5.18) that involve the phase angle. For a moderately large number of holes, we have $r_{k}^{\beta} \ll 1$, for $\beta \gg 1$, since $0<r_{k}<1$. Hence, the terms in (5.18) that involve $\Delta_{k, k^{\prime}}$ are numerically very small for any choice of the phase angles when there are a moderately large number of holes. In Fig. 8 we plot the optimum configurations corresponding to the data in Table 2.

Since the optimization leading to Table 2 is done only with respect to hole configurations satisfying (5.17), it is natural to ask whether one can obtain smaller values of $p$ for more general arrangements of holes in the unit disk. To study this question numerically, we used the routine fminunc of MATLAB to determine local minimum values of $p$ in (2.25) with respect to the $2 N$ variables $\left(x_{1}, y_{1}\right), \ldots,\left(x_{N}, y_{N}\right)$ denoting the hole locations within the unit disk. Since fminunc performs unconstrained nonlinear optimization, we constrained the holes to remain within the unit circle by setting $p$ to be very large for any $r_{i}=\sqrt{x_{i}^{2}+y_{i}^{2}} \geqslant 1$. We also provided the gradient and the Hessian matrix of $p$ as arguments to the optimization code. We used random locations of the $N$ holes in the unit circle as an initial guess. In some cases, the optimization code converged to different solutions depending on the initial condition. For each $N$, we performed multiple numerical 
Table 2. Numerical results for the optimum configuration within the class of two-ring and three-ring patterns of the form (5.17) with or without a centre hole. The first three columns indicate the optimum configuration, the minimum value of $p$, and the optimum ring radii. The last two columns correspond to the second best pattern. The notation $[1](5,12)$ indicates a two-ring pattern with a centre hole, which has 5 and 12 holes on the inner and outer rings, respectively

\begin{tabular}{rccccc}
\hline \hline & & & & \\
$N$ & Optimal pattern & $p_{\text {min }}$ & Optimum $r_{j}$ & Second best pattern & $p$ \\
\hline 6 & $(6)$ & -1.5260 & 0.642 & {$[1](5)$} & -1.5134 \\
7 & {$[1](6)$} & -1.8871 & 0.698 & $(7)$ & -1.8398 \\
8 & {$[1](7)$} & -2.2538 & 0.702 & $(2,6)$ & -2.1732 \\
9 & {$[1](8)$} & -2.6104 & 0.705 & $(2,7)$ & -2.5754 \\
10 & $(2,8)$ & -2.9686 & $0.222,0.737$ & $(1](9)$ & -2.9549 \\
11 & $(2,9)$ & -3.3498 & $0.212,0.736$ & $(2,10)$ & -3.3449 \\
12 & $(3,9)$ & -3.7546 & $0.288,0.760$ & $(4,9)$ & -3.7175 \\
13 & $(3,10)$ & -4.1511 & $0.277,0.758$ & $(3,11)$ & -4.1457 \\
14 & $(4,10)$ & -4.5660 & $0.327,0.776$ & $(5,10)$ & -4.5336 \\
15 & $(4,11)$ & -4.9728 & $0.316,0.773$ & $(4,12)$ & -5.3652 \\
16 & $(5,11)$ & -5.3903 & $0.354,0.788$ & {$[1](5,11)$} & -5.7921 \\
17 & $(5,12)$ & -5.8040 & $0.343,0.785$ & $(6,12)$ & -6.2195 \\
18 & {$[1](5,12)$} & -6.2242 & $0.408,0.797$ & {$[1](5,13)$} & -6.6422 \\
19 & {$[1](6,12)$} & -6.6713 & $0.429,0.809$ & {$[1](7,12)$} & -7.0983 \\
20 & {$[1](6,13)$} & -7.1052 & $0.418,0.805$ & {$[1](6,14)$} & -7.5257 \\
21 & {$[1](7,13)$} & -7.5480 & $0.436,0.815$ & {$[1](6,15)$} & -7.9313 \\
22 & {$[1](7,14)$} & -7.9844 & $0.426,0.811$ & $(2,8,15)$ & -8.4058 \\
23 & {$[1](8,14)$} & -8.4204 & $0.442,0.819$ & $(3,8,14)$ & -8.8561 \\
24 & {$[1](8,15)$} & -8.8566 & $0.433,0.816$ & -9.3020 \\
25 & $(2,8,15)$ & -9.3056 & $0.141,0.469,0.824$ & & \\
\hline \hline
\end{tabular}

realizations with random initial conditions to determine the minimum $p$-value of the local minima found by the code. We also ran the code using the theoretical results in Table 2 as initial data, where we used the optimal phase angle $\phi$ in (5.14). In Fig. 9 we show the optimization code results for the minimum $p$-value over at least 10 different random initial configurations. The dotted circular lines are the optimal ring radii of the $m$-ring configurations of Proposition 5.5.

For most configurations with two rings, the full optimization results yield hole locations that differ slightly from the configurations obtained by optimizing with respect to the two ring radii. This is not surprising, since we do not expect the minimization with respect to three variables (two radii and one angle) to yield a local minima of $p$, which is a function of $2 N$ variables. However, for $N \leqslant 25$, the local minima of $p$ in the two-ring case were found to be very close to the configurations obtained by minimizing over the radii and angles. In Figure 10 we compare the two minimization procedures for four different configurations. In all but one case, the optimization code converged to a pattern very similar to the theoretical results for ring patterns given in Table 2. 


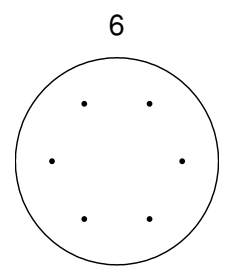

11

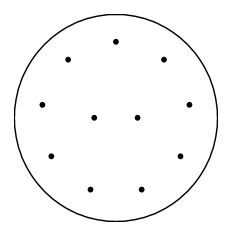

16

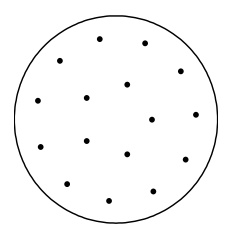

21

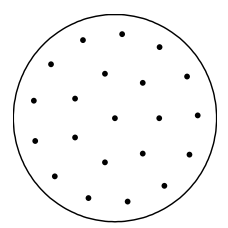

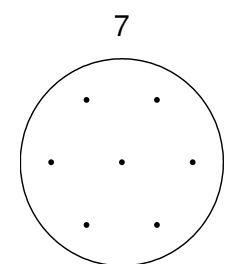

12

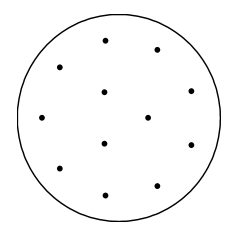

17

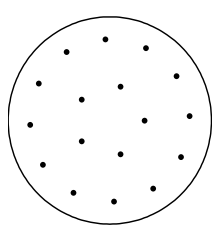

22

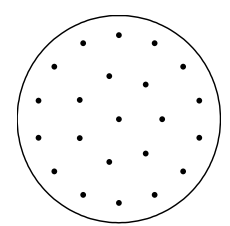

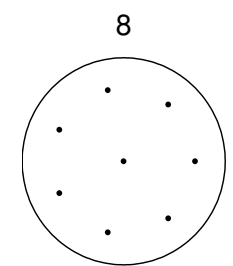

13

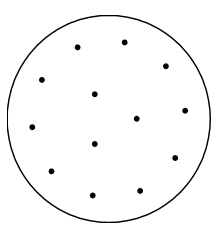

18

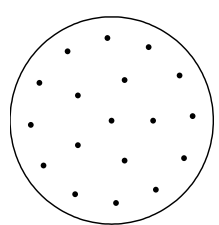

23

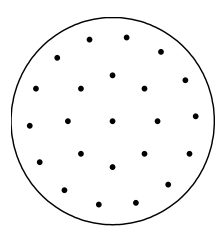

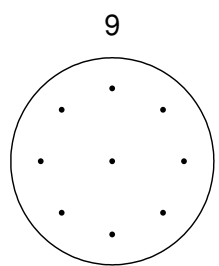

14

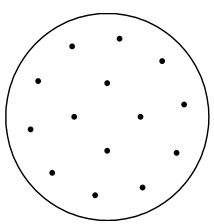

19

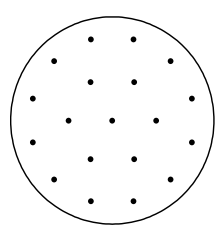

24

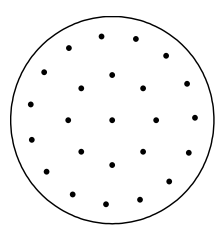

10

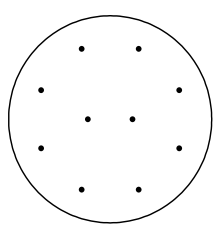

15

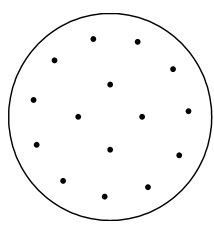

20

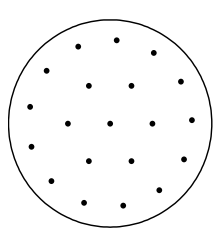

25

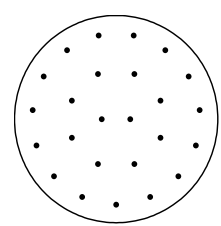

Figure 8. The optimum configurations for $N=6$ to $N=25$ holes within the class of two-ring and three-ring patterns, with or without a centre hole, given by Proposition 5.5. See Table 2.

In [9] the related discrete variational problem of minimizing $\mathscr{H}\left(x_{1}, \ldots, x_{N}\right)$, for $x_{j} \in \mathbb{R}^{2}$, was analyzed, where

$$
\mathscr{H}\left(x_{1}, \ldots, x_{N}\right)=-\frac{1}{2} \sum_{j=1}^{N} \sum_{\substack{k=1 \\ k \neq j}}^{N} \log \left|x_{j}-x_{k}\right|+\frac{1}{4} N(N-1) \log \left(\sum_{j=1}^{N}\left|x_{j}\right|^{2}\right) .
$$

The restricted optimization problem where $\mathscr{H}$ is minimized for particles placed on several rings, with and without a centre hole, was studied. For $N$ larger than certain threshold values these multi-ring solutions were found to be unstable with respect to the full optimization problem of minimizing $\mathscr{H}$ with respect to $2 N$ variables. However, the multiring patterns were still found to agree rather closely with the true minimum for $N=16$ and $N=21$ (see Fig. 2 of [9]). By comparing Fig. 8 and Fig. 9, and from Fig. 10, there is presumeably a similar qualitative relationship here between multi-ring optima of $p$ and the true optima computed from fminunc of MATLAB.

Next, we simplify (5.18) in the limit of a large number of holes. For $J_{1}, \ldots, J_{m} \gg 1$, we calculate the limiting stationary point for $p_{*}$ in $(5.18)$ by setting $\partial_{r_{k}} p_{*}=0$ for $k=1, \ldots, m$. 


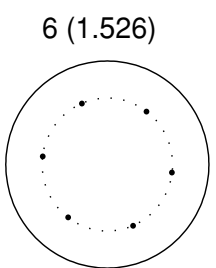

$11(3.3562)$

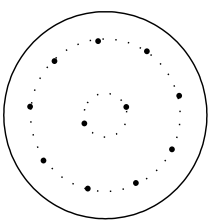

$16(5.3914)$

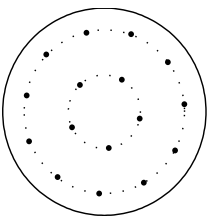

21 (7.5489)

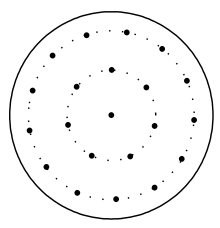

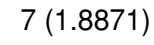

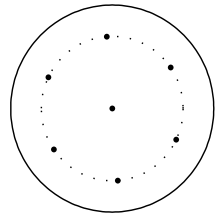

$12(3.7593)$

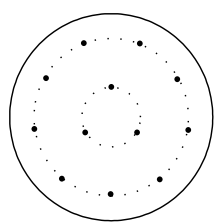

$17(5.8051)$

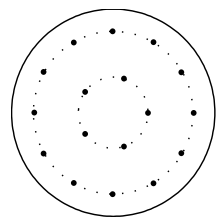

$22(7.985)$

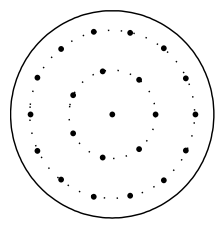

8 (2.2538)

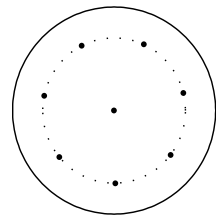

$13(4.1552)$

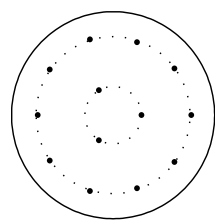

18 (6.2245)

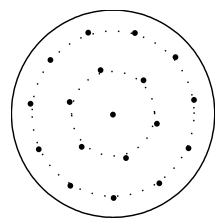

23 (8.4207)

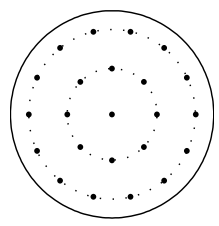

9 (2.6104)

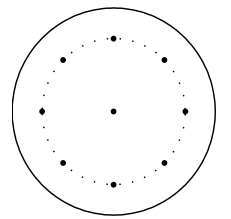

14 (4.5683)

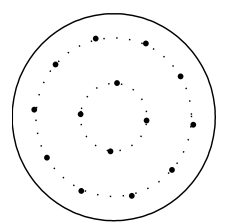

19 (6.6731)

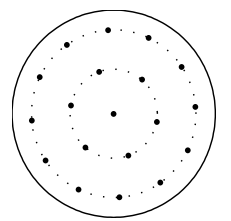

24 (8.8693)

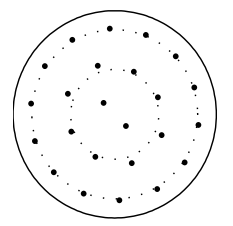

$10(2.976)$

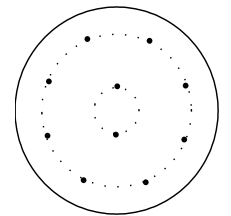

15 (4.975)

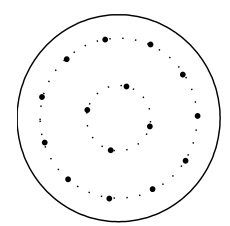

20 (7.1071)

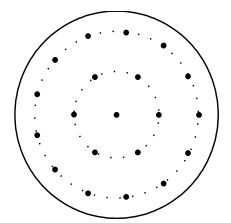

25 (9.3178)

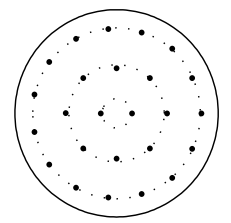

Figure 9. The optimum configurations for $N=6$ to $N=25$ holes computed using the routine fminunc of MATLAB. The values of $p$ for each pattern are given in the figure. The dotted circular lines are the optimal ring radii of the $m$-ring configurations of Proposition 5.5.

This leads to the following problem:

$$
\begin{aligned}
& -\frac{J_{1}^{2}}{r_{1}}+2 r_{1} J_{1}\left(J_{1}+\cdots+J_{m}\right)=0 ; \\
& -\frac{J_{q}^{2}}{r_{q}}+2 r_{q} J_{q}\left(J_{1}+\cdots+J_{m}\right)-\frac{2 J_{q}}{r_{q}} \sum_{p=1}^{q-1} J_{p}=0, \quad q=1, \ldots, m .
\end{aligned}
$$

For the case where $J_{1}=J_{2}=\ldots=J_{m} \gg 1$, we obtain from (5.21) that

$$
r_{q}=\sqrt{\frac{2(q-1)+1}{2 m}}, \quad q=1, \ldots, m .
$$

Since $r_{q+1}^{2}-r_{q}^{2}=1 / m$, this case corresponds to the partition of the unit disk into $m+1$ concentric areas $A_{q}: r_{q-1} \leqslant|x| \leqslant r_{q}$, for $q=1, \ldots, m+1$, where we have labelled $r_{0} \equiv 0$ and $r_{m+1}=1$. In this limit, we obtain $A_{1}=A_{m+1}=\pi /(2 m)$, and $A_{q}=\pi / m$ for $q=2, \ldots, m$. 

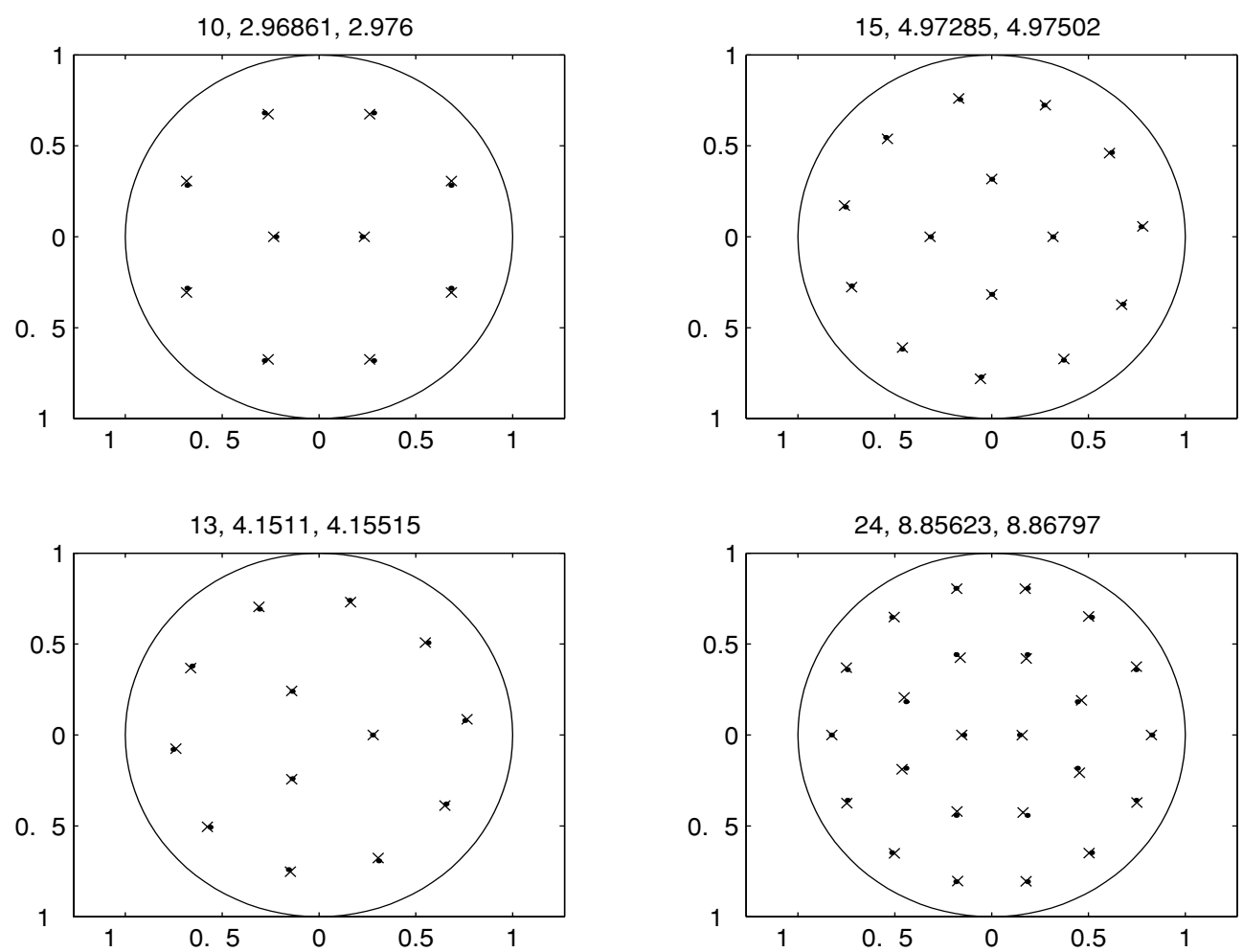

FIGURE 10. Comparison of the minimization of $p$ with respect to radii and angles (given by dots) and minimization with respect to all $2 N$ variables (given by crosses), obtained by using the dots as initial hole locations. The numbers in the title indicate the number of holes, and the two $p$ values, corresponding to the dots and crosses, respectively.

In the limit $J_{k} \gg 1$, but where the number of holes on each ring scales uniformly with the circumference of the ring, we obtain the following limiting result:

Proposition 5.6:(Limiting case of $\boldsymbol{m}$ rings) Consider an m-ring configuration with rings of radii $r_{1}, \ldots, r_{m}$. Assume that $J_{k} \gg 1$, but such that $J_{k}=\alpha r_{k}$ with $\alpha \gg 1$. Then, the $m$ rings partition the unit disk into $m+1$ concentric areas $A_{1}, \ldots, A_{m+1}$ with the property that $A_{q} / N_{q}$ is independent of $q$ for $q=1, \ldots, m+1$. Here $N_{q}$ is the number of holes on the boundary of the region $A_{q}$, with radius $r_{q}=q / \sqrt{m(m+1)}$.

Proof: Let $J_{q}=\alpha r_{q}$ in (5.21). Then, we obtain

$$
r_{q}+2 \sum_{\substack{p=1 \\ p<q}} r_{p}=2 r_{q}^{2} \zeta, \quad \zeta \equiv r_{1}+\cdots+r_{m}, \quad q=1, \ldots, m
$$

A simple calculation shows that $r_{q}=q /(2 \zeta)$. Therefore, $\zeta=\sum_{q=1}^{m} r_{q}$ yields $\zeta=$ $\sqrt{m(m+1)} / 2$ and $r_{q}=q / \sqrt{m(m+1)}$, for $q=1, \ldots, m$. Therefore, the ratio $A_{q} / N_{q}$ is 
independent of $q$ since

$$
\begin{aligned}
& \frac{A_{1}}{N_{1}}=\frac{\pi r_{1}^{2}}{J_{1}}=\frac{\pi r_{1}^{2}}{\alpha r_{1}}=\frac{\pi}{\alpha \sqrt{m(m+1)}}, \quad \frac{A_{m+1}}{N_{m+1}}=\frac{\pi\left(1-r_{m}^{2}\right)}{J_{m}}=\frac{\pi\left(1-r_{m}^{2}\right)}{\alpha r_{m}}=\frac{\pi}{\alpha \sqrt{m(m+1)}}, \\
& \frac{A_{q}}{N_{q}}=\frac{\pi\left(r_{q}^{2}-r_{q-1}^{2}\right)}{J_{q}+J_{q-1}}=\frac{\pi\left(r_{q}^{2}-r_{q-1}^{2}\right)}{\alpha\left(r_{q}+r_{q-1}\right)}=\frac{\pi\left(r_{q}-r_{q-1}\right)}{\alpha}=\frac{\pi}{\alpha \sqrt{m(m+1)}}, \quad q=2, \ldots, m .
\end{aligned}
$$

\section{Some related problems}

We now outline several different problems that are related to the minimization of $p\left(x_{1}, \ldots, x_{N}\right)$ in $(2.25)$.

The first application concerns an oxygen transport problem in a two-dimensional domain representing a transverse section of skeletal muscle tissue that receives oxygen from an array of capillaries of small cross-sectional area (cf. [27] and the references therein). Under certain simplifying assumptions (cf. [27]), the steady-state oxygen partial pressure distribution satisfies

$$
\Delta u=M, \quad x \in \Omega \backslash \Omega_{p} ; \quad \partial_{n} u=0, \quad x \in \partial \Omega ; \quad u=u_{c}, \quad x \in \partial \Omega_{p} .
$$

Here $\Omega_{p} \equiv \cup_{i=1}^{N} \Omega_{\varepsilon_{i}}$ denotes a collection of $N$ circular capillary cross-sections, each of radius $\varepsilon \ll 1$, that are centred at some $x_{i}$, for $i=1, \ldots, N$. The constant $M$ represents a spatially uniform oxygen consumption term, and $u_{c}$ is the oxygen partial pressure within each capillary, which is assumed to be constant for simplicity.

For $\varepsilon \ll 1,(6.1)$ has the same mathematical structure as that of the eigenvalue problem (1.1). Therefore, it can be solved asymptotically by the same technique as in $\S 2$. For $\varepsilon \ll 1$ and $\left|x-x_{i}\right| \gg O(\varepsilon)$ for $i=1, \ldots, N$, the outer solution for $u$ can be written in terms of the Neumann Green's function $G_{m}$ of (1.5) as (see $\S 4$ of [27])

$$
u(x ; \varepsilon) \sim u_{g}-2 \pi \sum_{j=1}^{N} A_{j} G_{m}\left(x ; x_{j}\right), \quad u_{g}=\bar{u} \equiv \frac{1}{|\Omega|} \int_{\Omega} u d x .
$$

As shown in [27], the constant $u_{g}$ and the singularity strengths $A_{j}$, for $j=1, \ldots, N$, in (6.2) satisfy the following coupled algebraic system in the limit $\varepsilon \rightarrow 0$ :

$$
\begin{aligned}
& A_{j}\left(1+2 \pi v R_{m}\left(x_{j} ; x_{j}\right)\right)+2 \pi v \sum_{\substack{k=1 \\
k \neq j}}^{N} A_{k} G_{m}\left(x_{j} ; x_{k}\right)=v\left(u_{g}-u_{c}\right), \quad j=1, \ldots, N, \\
& \sum_{k=1}^{N} A_{k}=-\frac{M|\Omega|}{2 \pi} .
\end{aligned}
$$

This system can be written in matrix form as

$$
\mathscr{C} \boldsymbol{a}=v \boldsymbol{e}_{0}\left(u_{g}-u_{c}\right), \quad \boldsymbol{e}_{0}^{t} \boldsymbol{a}=-\frac{M|\Omega|}{2 \pi} .
$$


Here $\mathscr{C}=I+2 \pi v \mathscr{G}$, where $\mathscr{G}$ is the $N \times N$ Green's function matrix defined in $(2.20 \mathrm{c})$. In addition, $\boldsymbol{e}_{0}^{t} \equiv(1, \ldots, 1), \boldsymbol{a}^{t} \equiv\left(A_{1}, \ldots, A_{N}\right)$, and $v=-1 / \log \varepsilon$. By solving (6.4) for $u_{g}$ and $\boldsymbol{a}$ we obtain

$$
u_{g}=u_{c}-\frac{M|\Omega|}{2 \pi v}\left[\boldsymbol{e}_{0}^{t} \mathscr{C}^{-1} \boldsymbol{e}_{0}\right]^{-1}, \quad \boldsymbol{a}=-\frac{M|\Omega|}{2 \pi} \frac{\mathscr{C}^{-1} \boldsymbol{e}_{0}}{\boldsymbol{e}_{0}^{t} \mathscr{C}^{-1} \boldsymbol{e}_{0}} .
$$

Recalling (6.2) for $u_{g}$, and using the asymptotic inverse $\mathscr{C}^{-1} \sim I-2 \pi \nu \mathscr{G}$ for $v \ll 1$, we obtain from (6.5) that

$$
\bar{u} \sim u_{c}-\frac{M|\Omega|}{2 \pi v N}-\left(\frac{M|\Omega|}{N^{2}}\right) p\left(x_{1}, \ldots, x_{N}\right)+O(v) .
$$

Here $p\left(x_{1}, \ldots, x_{N}\right)$, defined in (2.25), is the sum of all of the elements of the Green's function matrix $\mathscr{G}$.

From (6.6) we conclude that the average oxygen partial pressure $\bar{u}$ in the tissue is maximized, up to an $O(v)$ error term, when the capillary centres $x_{1}, \ldots, x_{N}$ are chosen to minimize the function $p$. This optimization problem is precisely the problem considered in $\S 3-\S 5$, and all of the results derived there can be applied directly.

Our second application concerns the determination of the equilibrium locations for spike solutions to the Gierer-Meinhardt reaction-diffusion system (cf. [8]) in two spatial dimensions given by

$$
a_{t}=\varepsilon^{2} \Delta a-a+\frac{a^{2}}{h}, \quad x \in \partial \Omega ; \quad 0=D \Delta h-h+\frac{a^{2}}{\varepsilon^{2}}, \quad x \in \partial \Omega,
$$

with $\partial_{n} a=0$ and $\partial_{n} h=0$ on $\partial \Omega$. Localized solutions in the activator concentration $a$, which have a spatial support of $O(\varepsilon)$, can be constructed using the method of matched asymptotic solutions. Such solutions are called spike solutions. For $\varepsilon \ll 1$ and for $D \gg O(1)$, and assuming that the spike profile is stable, the locations $x_{j}$ of a collection of $N$ spikes of the same height are found to satisfy (cf. [14])

$$
\frac{d x_{j}}{d t} \sim-\frac{4 \pi \varepsilon^{2} v}{1+2 \pi v D N|\Omega|^{-1}}\left(\left.\nabla R_{m}\left(x ; x_{j}\right)\right|_{x=x_{j}}+\left.\sum_{\substack{k=1 \\ k \neq j}}^{N} \nabla G_{m}\left(x ; x_{k}\right)\right|_{x=x_{j}}\right) .
$$

Here $G_{m}$ and $R_{m}$ are, again, the Neumann Green's functions defined in (1.5). The case of a one-spike solution, where the equilibrium spike is located at a zero of $\nabla R_{m}\left(x_{0} ; x_{0}\right)=0$, was studied in $[15,16]$.

By comparing (6.8) with (2.25), it is clear that stable equilibrium spike locations under the flow (6.8) correspond to minimum points of the function $p\left(x_{1}, \ldots, x_{N}\right)$ in (2.25). Hence, the analysis given in $\S 3-\S 5$ for the minima of $p$ for certain configurations of $x_{1}, \ldots, x_{N}$ correspond to stable equilibrium spike locations with respect to (6.8). For the corresponding Gierer-Meinhardt shadow system, where $D=\infty$ in (6.7), it is well-known (cf. $[2,10]$ ) that the equilibrium spike locations in a convex domain are determined not by minimizing $p\left(x_{1}, \ldots, x_{N}\right)$ but, instead, by the geometric problem of the packing of balls of equal radii inside the domain. 
Next, we consider a ring-like solution of the Gray-Scott model in a two-dimensional disk. Such solutions have been recently analyzed in [17, 18, 22]. This reaction-diffusion model can be written as (cf. [17])

$$
v_{t}=\varepsilon^{2} \Delta v-v+A u v^{2}, \quad \tau u_{t}=\Delta u-u+u v^{2}, \quad x \in D_{R}=\{x:|x|<R\},
$$

with homogeneous Neumann boundary conditions at $x=R$. For $\varepsilon \ll 1$, and for the range $O\left(\varepsilon^{1 / 2}\right) \ll A \ll O(1)$, it was shown in [18, 22] that there is an equilibrium ring-like solution of the form $v(x) \sim C w\left[\varepsilon^{-1}(|x|-\rho)\right]$ and $u(x) \sim u_{0}$, where $w(r)=\frac{3}{2} \operatorname{sech}^{2}(r / 2)$. Here $C$, $u_{0}$ are some constants, and the ring radius $\rho$ depends only on the disk radius $R$. Explicit expressions for these constants were derived in [18, 22]. For $R \ll 1$, it was found in [17] that $\rho \sim R / \sqrt{2}$. Therefore, the ring radius is such that the unit disk is divided into two equal parts. Recall that this geometrical description also applies to optimizing the radius of a single ring of holes in the limit $N \rightarrow \infty$ (see the discussion after Proposition 4.7 and Proposition 5.6) for the singularly perturbed eigenvalue problem. A deeper geometrical connection between these two limiting results is desirable.

Finally, we relate our problem of optimal hole locations for (1.1) in the unit disk to the problem of determining equilibrium vortex configurations within the context of the Ginzburg-Landau theory of superconductivity. For the unit disk, a little algebra shows that the minimization of $p$ in (2.25) is equivalent to the problem of minimizing the function $\mathscr{F}\left(x_{1}, \ldots, x_{N}\right)$ defined by

$$
\mathscr{F}\left(x_{1}, \ldots, x_{N}\right)=-\sum_{j=1}^{N} \sum_{\substack{k=1 \\ k \neq j}}^{N} \log \left|x_{j}-x_{k}\right|-\sum_{j=1}^{N} \sum_{k=1}^{N} \log \left|1-x_{j} \bar{x}_{k}\right|+N \sum_{j=1}^{N}\left|x_{j}\right|^{2}, \quad\left|x_{j}\right|<1,
$$

for $x_{j} \neq x_{k}$ when $j \neq k$. In contrast, upon taking a certain limit of a variational formulation of the Ginzburg-Landau model of superconductivity, it was shown in [21, 25] that for an equilibrium vortex configuration $x_{1}, \ldots, x_{N}$ within the unit disk, with vortices of a common winding number, the vortex locations correspond to a minimum point of the renormalized energy $\mathscr{W}\left(x_{1}, \ldots, x_{N}\right)$ defined by

$$
\mathscr{W}\left(x_{1}, \ldots, x_{N}\right)=-\sum_{j=1}^{N} \sum_{\substack{k=1 \\ k \neq j}}^{N} \log \left|x_{j}-x_{k}\right|-\sum_{j=1}^{N} \sum_{k=1}^{N} \log \left|1-x_{j} \bar{x}_{k}\right|, \quad\left|x_{j}\right|<1 .
$$

This energy is precisely the first two terms in (6.10). Therefore, the optimal hole location problem and the vortex configuration problem are indeed very similar and differ only by the 'confinement potential' term $N \sum_{j=1}^{N}\left|x_{j}\right|^{2}$ in (6.10). An optimal two-vortex solution for (6.11) was constructed in [21]. By taking the continuum limit $N \rightarrow \infty$ in (6.11), it was proved in [25] that for a minimizing configuration $x_{1}^{N}, \ldots, x_{N}^{N}$ of $N$ vortices, the measures $\mu_{N}=\frac{1}{N} \sum_{j=1}^{N} \delta\left(x-x_{j}^{N}\right)$ converge weakly to the uniform measure on the boundary $\partial \Omega$ of the unit disk $\Omega$. For $N \rightarrow \infty$, the effect of the confinement potential in (6.10) should be to equi-distribute the optimal hole locations within the unit disk. Therefore, for $N \rightarrow \infty$, the limiting optimal configuration of hole locations for (6.10) will not correspond to a 
multi-ring pattern of holes. As a remark, for vortices in superconductivity with different integer winding numbers $d_{1}, \ldots, d_{N}$, the renormalized energy in (6.11) is replaced by

$$
\mathscr{W}\left(x_{1}, \ldots, x_{N}\right)=-\sum_{j=1}^{N} \sum_{\substack{k=1 \\ k \neq j}}^{N} d_{j} d_{k} \log \left|x_{j}-x_{k}\right|-\sum_{j=1}^{N} \sum_{k=1}^{N} d_{j} d_{k} \log \left|1-x_{j} \bar{x}_{k}\right|, \quad\left|x_{j}\right|<1 .
$$

This weighted minimization problem is closely related to the problem of maximizing the first eigenvalue, given in (2.22) of Proposition 2.2, for (1.1) where the $N$ holes have different shapes.

\section{Conclusion}

We have given some analytical and numerical results for the optimization of the fundamental eigenvalue $\lambda_{0}$ of (1.1) with respect to the locations of $N$ small traps in a two-dimensional domain. This optimization problem involves the minimization of the function $p\left(x_{1}, \ldots, x_{N}\right)$ in (2.25). The problem of minimizing $p\left(x_{1}, \ldots, x_{N}\right)$ also arises in several diverse settings, including, minimizing the expected lifetime of Brownian motion in a domain with reflecting walls, maximizing the average oxygen partial pressure in a cross-section of muscle tissue containing capillary cross-sections, and determining stable equilibrium spike locations for a collection of $N$ spikes for the two-dimensional GiererMeinhardt model. The problem of minimizing $p\left(x_{1}, \ldots, x_{N}\right)$ is also closely related to the problem of determining equilibrium vortex configurations in the Ginzburg-Landau theory of superconductivity.

For the unit disk, where the Neumann Green's function can be calculated analytically, rather precise results have been given for the minimization of $p\left(x_{1}, \ldots, x_{N}\right)$ for certain ring-type configurations of traps. For $N \gg 1$ traps on one ring, the optimal ring radius was found to geometrically divide the unit disk into two regions of the same area. This limiting behaviour is also what was found in [17] for the equilibrium construction of a ring solution for the Gray-Scott model in a certain parameter regime.

For the case of one trap in an asymmetric dumbbell-shaped domain, we have shown that the location of a trap corresponding to a local maximum of $\lambda_{0}$ is not necessarily unique. A key open problem is to determine conditions on the domain that ensure the uniqueness of the root to $\nabla R_{m 0}=0$. The problem of maximizing $\lambda_{0}$ for (1.1) with respect to the locations of $N$ traps in other domains such as the unit square, where there is no simple representation of the Neumann Green's function, is also open.

\section{Acknowledgements}

T. K. was supported by a PGS-B graduate scholarship from NSERC (Canada). M. S. T. was supported by a CRM-ISM Postdoctoral Fellowship in Montreal. M. J. W. was supported by NSERC under grant 81541 . We would like to thank Prof. Juncheng Wei of the Chinese U. of Hong Kong for a few helpful discussions. We would like to thank an anonymous reviewer for some insightful comments regarding Ginzburg-Landau vortex configurations. 


\section{References}

[1] Bandle, C. \& Flucher, M. (1996) Harmonic radius and concentration of energy; Hyperbolic radius and Liouville's equations $\triangle U=e^{U}$ and $\triangle U=U^{(n+2) /(n-2)}$. SIAM Rev. 38(2), 191-238.

[2] Bates, P. \& Fusco, N. (2000) Equilibria with many nuclei for the Cahn-Hilliard equation. J. Diff. Eq. 160(2), 283-356.

[3] Caffarelli, L. A. \& Friedman, A. (1985) Convexity of solutions of semilinear Elliptic equations. Duke Math. J. 52(2), 431-456.

[4] Chen, X. \& KowalczyK, M. (2001) Dynamics of an interior spike in the Gierer-Meinhardt system. SIAM J. Math. Anal. 33(1), 172-193.

[5] Cox, S. J. \& Uhlig, P. X. (1999) Where best to hold a drum fast. SIAM J. Optimization 9(4), 948-964.

[6] Denzler, J. (1999) Windows of given area with minimal heat diffusion. Trans. Amer. Math. Soc. 351(2), 569-580.

[7] Flucher, M. (1995) Approximation of Dirichlet Eigenvalues on domains with small holes. J. Math. Anal. Appl. 193(1), 169-199.

[8] Gierer, A. \& Meinhardt, H. (1972) A theory of biological pattern formation. Kybernetik 12, 30-39.

[9] Gueron, S. \& Shafrir, I. (1999) On a discrete variational problem involving interacting particles. SIAM J. Appl. Math. 60(1), 1-17.

[10] GuI, C. \& WeI, J. (1999) Multiple interior peak solutions for some singularly Perturbed Neumann problems. J. Diff. Eq. 158(1), 1-27.

[11] Gustafsson, B. (1990) On the convexity of a solution of Liouville's equation. Duke Math. J. 60(2), 303-311.

[12] Harrell II, E. M., Kröger, P. \& Kurata, K. (2001) On the placement of an obstacle or a well so as to optimize the fundamental eigenvalue. SIAM J. Math. Anal. 33(1), 240-259.

[13] Henrot, A. (2003) Minimization problems for Eigenvalues of the Laplacian. J. Evol. Equ. 3(3), 443-461.

[14] Kolokolnikov, T. (2004) unpublished notes.

[15] Kolokolnikov, T. \& Ward, M. J. (2003) Reduced Wave Green's functions and their effect on the dynamics of a spike for the Gierer-Meinhardt model. Europ. J. Appl. Math. 14(5), $513-545$.

[16] Kolokolnikov, T. \& Ward, M. J. (2004) Bifurcation of spike equilibria in the near-shadow Gierer-Meinhardt model. Discrete Cont. Dyn. Sys. Series B 4(4), 1033-1064.

[17] Kolokolnikov, T., Ward, M. J. \& WeI, J. (2005) Zigzag and breakup instabilities of stripes and rings in the two-dimensional Gray-Scott model, submitted, Studies in Appl. Math.

[18] Kolokolnikov, T. \& Wei, J. (2004) On Ring-Like solutions for the Gray-Scott Model: Existence, instability and self-replicating rings, accepted. Europ. J. Appl. Math.

[19] LaI, Ying-Ju. \& Lin, I (1999) Packings and defects of strongly coupled two-dimensional coulomb clusters: Numerical simulation, Phys. Rev. E. 60(4), 4743-4753.

[20] Lange, C. \& Weinitschke, H. (1994) Singular perturbations of elliptic problems on domains with small holes. Stud. Appl. Math. 92(1), 55-93.

[21] Lefter, C. \& Radulescu, V. (1996) Minimization problems and corresponding renormalized energies. Differential Integral Equations 9(5), 903-917.

[22] Morgan, D. \& KaPER, T. (2004) Axisymmetric ring solutions of the 2-D Gray-Scott model and their destabilization into spots. Physica D 192(1-2), 33-62.

[23] Ozawa, S. (1981) Singular variation of domains and eigenvalues of the Laplacian. Duke Math. J. 48(4), 767-778.

[24] Saint Jean, M., Even, C. \& Guthmann, C. (2001) Macroscopic 2D Wigner Islands. Europhys. Lett. 55(1), 45-51.

[25] SANdier, E. \& Soret, M. (2000) $S^{1}$-Valued harmonic maps with high topological degree: Asymptotic behavior of the singular set, Potential Analysis 13(2), 169-184. 
[26] Swanson, C. A. (1963) Asymptotic variational formulae for eigenvalues. Canad. Math. Bull. 6, $15-25$.

[27] Titcombe, M. S. \& Ward, M. J. (2000) An asymptotic study of oxygen transport from multiple capillaries to skeletal muscle tissue, SIAM J. Appl. Math. 60(5), 1767-1788.

[28] Ward, M. J., Henshaw, W. D. \& Keller, J. (1993) Summing logarithmic expansions for singularly perturbed eigenvalue problems, SIAM J. Appl. Math. 53(3), 799-828.

[29] Ward, M. J. \& Keller, J. B. (1993) Strong localized perturbations of eigenvalue problems. SIAM J. Appl. Math. 53(3), 770-798.

[30] Ward, M. J., McInerney, D., Houston, P., Gavaghan, D. \& Maini, P. (2002) The dynamics and pinning of a spike for a reaction-diffusion system. SIAM J. Appl. Math. 62(4), 1297-1328. 